The Astrophysical Journal, 689:1044-1062, 2008 December 20

(C) 2008. The American Astronomical Society. All rights reserved. Printed in U.S.A.

\title{
MEASURING DISTANCE AND PROPERTIES OF THE MILKY WAY'S CENTRAL SUPERMASSIVE BLACK HOLE WITH STELLAR ORBITS
}

\author{
A. M. Ghez, ${ }^{1,2}$ S. Salim, ${ }^{1,3}$ N. N. Weinberg, ${ }^{4,5}$ J. R. Lu, ${ }^{1}$ T. Do, ${ }^{1}$ J. K. Dunn, ${ }^{1}$ K. Matthews, ${ }^{4}$ M. R. Morris, ${ }^{1}$ \\ S. Yelda, ${ }^{1}$ E. E. Becklin, ${ }^{1}$ T. Kremenek, ${ }^{1}$ M. Milosavluevic, ${ }^{6}$ and J. Naiman ${ }^{1,7}$ \\ Received 2008 June 17; accepted 2008 August 20
}

\begin{abstract}
We report new precision measurements of the properties of our Galaxy's supermassive black hole. Based on astrometric (1995-2007) and radial velocity (RV; 2000-2007) measurements from the W. M. Keck $10 \mathrm{~m}$ telescopes, a fully unconstrained Keplerian orbit for the short-period star S0-2 provides values for the distance $\left(R_{0}\right)$ of $8.0 \pm 0.6 \mathrm{kpc}$, the enclosed mass $\left(M_{\mathrm{bh}}\right)$ of $4.1 \pm 0.6 \times 10^{6} M_{\odot}$, and the black hole's RV, which is consistent with zero with $30 \mathrm{~km} \mathrm{~s}^{-1}$ uncertainty. If the black hole is assumed to be at rest with respect to the Galaxy (e.g., has no massive companion to induce motion), we can further constrain the fit, obtaining $R_{0}=8.4 \pm 0.4 \mathrm{kpc}$ and $M_{\mathrm{bh}}=4.5 \pm 0.4 \times 10^{6} M_{\odot}$. More complex models constrain the extended dark mass distribution to be less than $3-4 \times 10^{5} M_{\odot}$ within $0.01 \mathrm{pc}, \sim 100$ times higher than predictions from stellar and stellar remnant models. For all models, we identify transient astrometric shifts from source confusion (up to 5 times the astrometric error) and the assumptions regarding the black hole's radial motion as previously unrecognized limitations on orbital accuracy and the usefulness of fainter stars. Future astrometric and RV observations will remedy these effects. Our estimates of $R_{0}$ and the Galaxy's local rotation speed, which it is derived from combining $R_{0}$ with the apparent proper motion of Sgr A*, $\left(\theta_{0}=229 \pm 18 \mathrm{~km} \mathrm{~s}^{-1}\right)$, are compatible with measurements made using other methods. The increased black hole mass found in this study, compared to that determined using projected mass estimators, implies a longer period for the innermost stable orbit, longer resonant relaxation timescales for stars in the vicinity of the black hole and a better agreement with the $M_{\mathrm{bh}}-\sigma$ relation.
\end{abstract}

Subject headings: black hole physics — Galaxy: center — Galaxy: kinematics and dynamics — infrared: stars techniques: high angular resolution

\section{INTRODUCTION}

Ever since the discovery of fast-moving $\left(v>1000 \mathrm{~km} \mathrm{~s}^{-1}\right)$ stars within $0.3^{\prime \prime}(0.01 \mathrm{pc})$ of our Galaxy's central supermassive black hole (Eckart \& Genzel 1997; Ghez et al. 1998), the prospect of using stellar orbits to make precision measurements of the black hole's mass $\left(M_{\mathrm{bh}}\right)$ and kinematics, the distance to the Galactic center $\left(R_{0}\right)$, and, more ambitiously, to measure postNewtonian effects has been anticipated (Jaroszyński 1998, 1999; Salim \& Gould 1999; Fragile \& Mathews 2000; Rubilar \& Eckart 2001; Weinberg et al. 2005; Zucker \& Alexander 2007; Kraniotis 2007; Will 2008). An accurate measurement of the Galaxy's central black hole mass is useful for putting the Milky Way in context with other galaxies through the apparent relationship between the mass of the central black hole and the velocity dispersion, $\sigma$, of the host galaxy (e.g., Ferrarese \& Merrit 2000; Gebhardt et al. 2000; Tremaine et al. 2002). It can also be used as a test of this scaling, as the Milky Way has the most convincing case for a supermassive black hole of any galaxy used to define this relationship. Accurate estimates of $R_{0}$ impact a wide range

\footnotetext{
1 Department of Physics and Astronomy, UCLA, Los Angeles, CA 90095-1547; ghez@astro.ucla.edu,jlu@astro.ucla.edu,tdo@astro.ucla.edu,jkdunn@astro.ucla .edu, morris@astro.ucla.edu, syelda@astro.ucla.edu, becklin@astro.ucla.edu.

${ }^{2}$ Institute of Geophysics and Planetary Physics, UCLA, Los Angeles, CA 90095-1565.

3 National Optical Astronomy Observatory, 950 North Cherry Avenue, Tucson, AZ 85719; samir@noao.edu.

4 Division of Mathematics, Physics and Astronomy, California Institute of Technology, Pasadena, CA 91125; kym@caltech.edu.

5 Department of Astronomy, University of California, Berkeley, CA 947203411; nnw@astron.berkeley.edu.

6 Department of Astronomy, University of Texas, Austin, TX 78712; milos (a)astro.as.utexas.edu.

7 Department of Astronomy and Astrophysics, University of California, Santa Cruz, CA 95064; jnaiman@astro.ucsc.edu.
}

of issues associated with the mass and structure of the Milky Way, including possible constraints on the shape of the dark matter halo and the possibility that the Milky Way is a lopsided spiral (e.g., Reid 1993; Olling \& Merrifield 2000; Majewski et al. 2006). Furthermore, if measured with sufficient accuracy $(\sim 1 \%)$, the distance to the Galactic center could influence the calibration of standard candles, such as RR Lyrae stars, Cepheid variables, and giants, used in establishing the extragalactic distance scale. In addition to estimates of $M_{\mathrm{bh}}$ and $R_{0}$, precision measurements of stellar kinematics offer the exciting possibility of detecting deviations from a Keplerian orbit. This would allow an exploration of a possible cluster of stellar remnants surrounding the central black hole, suggested by Morris (1993), Miralda-Escudé \& Gould (2000), and Freitag et al. (2006). Estimates for the mass of the remnant cluster range from $10^{4}-10^{5} M_{\odot}$ within a few tenths of a parsec of the central black hole. Absence of such a remnant cluster would be interesting in view of the hypothesis that the inspiral of intermediate-mass black holes by dynamical friction could deplete any centrally concentrated cluster of remnants. Likewise, measurements of post-Newtonian effects would provide a test of general relativity, and ultimately could probe the spin of the central black hole.

Tremendous observational progress has been made over the last decade toward obtaining accurate estimates of the orbital parameters of the fast-moving stars at the Galactic center. Patience alone permitted new astrometric measurements that yielded the first accelerations (Ghez et al. 2000; Eckart et al. 2002), which suggested that the orbital period of the best characterized star, $\mathrm{S} 0-2$, could be as short as $15 \mathrm{yr}$. The passage of more time then led to full astrometric orbital solutions (Schödel et al. 2002, 2003; Ghez et al. 2003, 2005a), which increased the implied dark mass densities by a factor of $10^{4}$ compared to earlier velocity dispersion work and thereby solidified the case for a supermassive black 
hole. The advent of adaptive optics enabled radial velocity (RV) measurements of these stars (Ghez et al. 2003), which permitted the first estimates of the distance to the Galactic center from stellar orbits (Eisenhauer et al. 2003, 2005).

In this paper we present new orbital models for S0-2. These provide the first estimates of the distance to the Galactic center and limits on the extended mass distribution based on data collected with the W. M. Keck telescopes. The ability to probe the properties of the Galaxy's central supermassive black hole has benefitted from several advances since our previous report (Ghez et al. 2005a). First, new astrometric and RV measurements have been collected between 2004 and 2007, increasing the quantity of kinematic data available. Second, the majority of the new data was obtained with the laser guide-star adaptive optics system at Keck, improving the quality of the measurements (Ghez et al. 2005b; Hornstein et al. 2007). These new data sets are presented in $\S 2$. Finally, new data analysis has improved our ability to extract RV estimates from past spectroscopic measurements, allowing us to extend the RV curve back in time by $2 \mathrm{yr}$, as described in $\S 3$. The orbital analysis, described in $\S 4$, identifies several sources of previously unrecognized biases and the implications of our results are discussed in $\S 5$.

\section{OBSERVATIONS AND DATA SETS}

\subsection{High Angular Resolution Imaging: Speckle and Adaptive Optics}

For the first 11 yr of this experiment (1995-2005), the proper motions of stars orbiting the center of our Galaxy were obtained from $K[2.2 \mu \mathrm{m}]$-band speckle observations of the central stellar cluster with the W. M. Keck I 10 m telescope and its facility near-infrared camera (NIRC; Matthews \& Soifer 1994; Matthews et al. 1996). A total of 27 epochs of speckle observations are included in the analysis conducted in this paper, of which 22 have been reported in earlier papers by our group (Ghez et al. 1998, 2000, 2005a). Five new speckle observations, between 2004 April and 2005 June, were conducted in a similar manner. In summary, during each observing run, $\sim 10,000$ short $\left(t_{\text {exp }}=0.1 \mathrm{~s}\right)$ exposure frames were obtained with NIRC in its fine plate scale mode, which has a scale of $20.46 \pm 0.01$ mas pixel $^{-1}$ (see Appendix B) and a corresponding field of view of $5.2^{\prime \prime} \times 5.2^{\prime \prime}$. Interleaved with these observations were similar sequences on a dark patch of sky. From these data, we produce images that are diffraction limited $\left(\theta=0.05^{\prime \prime}\right)$ and have Strehl ratios of $\sim 0.05$.

With the advent of laser guide-star adaptive optics (LGSAO) in 2004 on the $10 \mathrm{~m} \mathrm{~W}$. M. Keck II telescope (Wizinowich et al. 2006; van Dam et al. 2006), we have made measurements of the Galaxy's central stellar cluster with much higher Strehl ratios (Ghez et al. 2005b). Between 2004 and 2007, nine LGSAO data sets were taken using the W. M. Keck II facility near-infrared camera, NIRC2 (PI: K. Matthews), which has an average plate scale of $9.963 \pm 0.006$ mas pixel $^{-1}$ (see Appendix C) and a field of view of $10.2^{\prime \prime} \times 10.2^{\prime \prime}$. All but one of the observations were obtained through a $K^{\prime}\left(\lambda_{0}=2.12 \mu \mathrm{m}, \Delta \lambda=0.35 \mu \mathrm{m}\right)$ bandpass filter, with the remaining one obtained through narrowband filters (CO: $\lambda_{0}=2.278 \mu \mathrm{m}, \Delta \lambda=0.048 \mu \mathrm{m}$; and $K_{\text {cont }}: \lambda_{0}=2.27 \mu \mathrm{m}$, $\Delta \lambda=0.030 \mu \mathrm{m})$. During these observations, the laser guidestar's position was fixed to the center of the camera's field of view and therefore moved when the telescope was dithered. While the laser guide star is used to correct most of the important atmospheric aberrations, it does not provide information on the tip-tilt term, which, for all our LGSAO observations (imaging and spectroscopy), was obtained from visible observations of USNO 0600$28577051\left(R=13.7 \mathrm{mag}\right.$ and $\left.\Delta r_{\mathrm{SgrA}^{*}}=19^{\prime \prime}\right)$. Details of the observing setup for 2004 July 26, 2005 June 30, and 2005 July 31 are described in detail in Ghez et al. (2005b), Lu et al. (2008), and Hornstein et al. (2007), respectively. While each of these early LGSAO observations had a slightly different setup and dither pattern, the more recent, deeper, LGSAO measurements (20062007) were obtained with nearly identical setups. Specifically, we used a 20-position dither pattern with randomly distributed (but repeatable) positions in a $0.7^{\prime \prime} \times 0.7^{\prime \prime}$ box and an initial position that placed IRS $16 \mathrm{NE}$ on pixel $(229,720)$ at a sky position angle (P.A.) set to 0.0 . This setup keeps the brightest star in the region, IRS $7(K=6.4)$, off the field of view at all times. At each position, three exposures, each composed of 10 co-added $2.8 \mathrm{~s}$ integrations, were obtained; the integration time was set with the aim of keeping the detector's response linear beyond the full width at half maximum (FWHM) point for the brightest $(K=9.0)$ star in the field of view; the number of images per position was chosen to provide the minimum elapsed time needed to allow the LGSAO system's optimization algorithm to converge ( $\sim 3$ minutes) before dithering. Table 1 summarizes all the new imaging data sets.

\subsection{Adaptive Optics Spectroscopy}

To monitor the line-of-sight motions of stars orbiting the center of our Galaxy between the years 2000 and 2007, high angular resolution spectroscopic observations of stars in the Sgr A* stellar cluster were taken with both the natural guide star adaptive optics (NGSAO; Wizinowich et al. 2000) system (2000-2004) and the LGSAO system (2005-2007) on the W. M. Keck II 10 m telescope. The NGSAO atmospheric corrections and the LGSAO tip-tilt corrections were made on the basis of visible observations of USNO 0600-28579500 ( $R=13.2 \mathrm{mag}$ and $\left.\Delta r \sim 30^{\prime \prime}\right)$ and USNO 0600-28577051 ( $R=13.7 \mathrm{mag}$ and $\left.\Delta r \sim 19^{\prime \prime}\right)$, respectively. While the angular resolution of the NGSAO spectra was typically $2-3$ times the diffraction limit $\left(\theta_{\text {diff }}=54\right.$ mas $)$, a pointspread function (PSF) FWHM of $\sim 70$ mas at $2 \mu \mathrm{m}$ was achieved for the LGSAO long-exposure spectra.

Three different spectrometers have been used over the course of this study. Our earliest measurements were obtained in 2000 June with NIRSPEC (McLean et al. 1998, 2000) in its low-resolution slit spectrometer mode $(R \sim 2600)$. It was not originally designed to go behind the adaptive optics system and therefore had inefficient throughput in its AO mode; it was, however, the only spectrometer available behind the AO system in 2000. While the resulting low signal-to-noise ratio $(\mathrm{S} / \mathrm{N})$ data set yielded no line detections in the initial analysis of S0-2 (Gezari et al. 2002), we now have the advantage of knowing what type of lines are present in the spectra and have therefore included this data set in our analysis by retroactively identifying the $\mathrm{Br} \gamma$ line, which is used to measure radial velocities (see $\S 3.2$ ). Between 2002 and 2005, NIRC2 (PI: K. Matthews) was used in its spectroscopic $R \sim 4000$ mode, which is generated with a 20 mas pixel scale, a mediumresolution grism, and a 2 pixel slit. In 2002 this produced the first line detection in S0-2 (Ghez et al. 2003), and since then three new NIRC2 measurements (two with NGSAO and one with LGSAO) have been obtained. Since 2005 OSIRIS, which is an integral field spectrograph with a $2 \mu \mathrm{m}$ spectral resolution of $\sim 3600$ (Larkin et al. 2006) has been used. The field of view of this spectrograph depends on the pixel scale and filter. Most of the OSIRIS observations were taken using the 35 mas pixel scale and the narrowband filter $\mathrm{Kn} 3$ (2.121 to $2.229 \mu \mathrm{m}$; includes $\mathrm{Br} \gamma$ ), which results in a field of view of $1.12^{\prime \prime} \times 2.24^{\prime \prime}$, and were centered on S0-2. All of the OSIRIS observations were obtained with the LGSAO system. Table 2 summarizes the details of the 10 new spectroscopic measurements of S0-2 that were made between the years 
TABLE 1

Summary of New Keck Imaging Observations

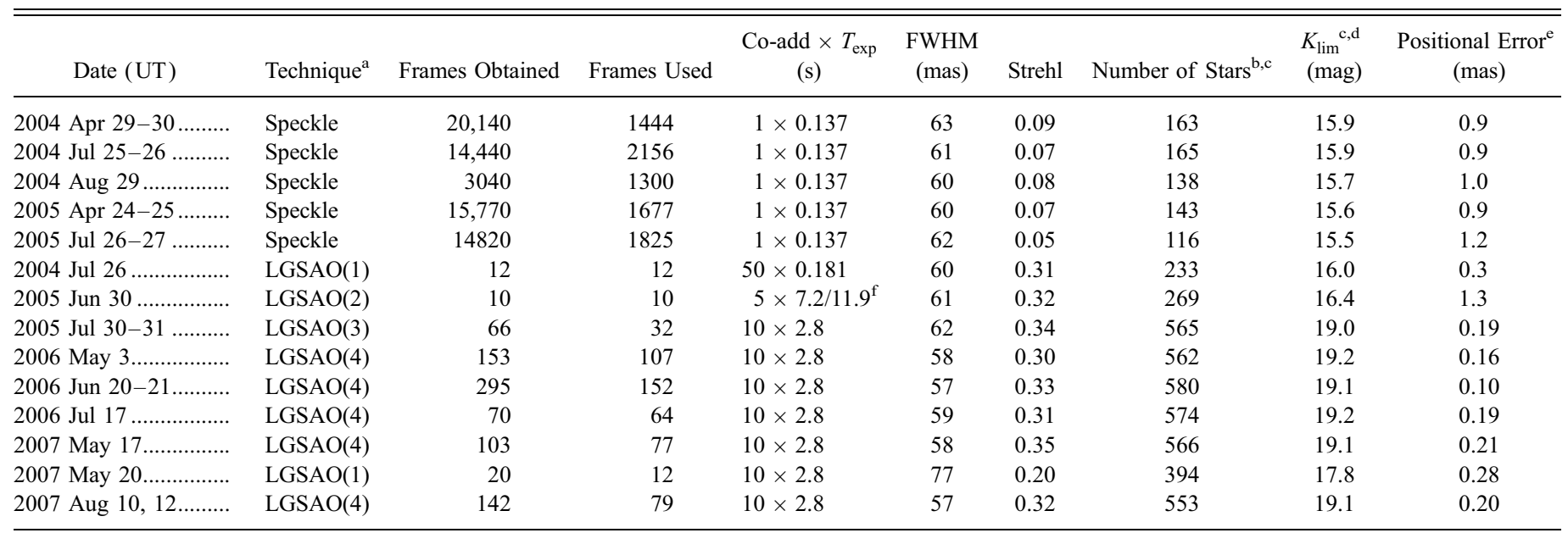

${ }^{a}$ For the LGSAO data sets, the number in parentheses denotes the observational setup used (e.g., dither pattern and camera orientation; see $\S 2.1$ for details).

b The number of stars detected within $3^{\prime \prime}$ of Sgr A*.

${ }^{c}$ For this analysis only stars in four or more epochs are considered to eliminate any spurious source detections.

${ }^{\mathrm{d}} K_{\lim }$ is the magnitude at which the cumulative distribution function of the observed $K$ magnitudes reaches $90 \%$ of the total sample size.

e The average positional uncertainty due to centroiding in each epoch is estimated from a set of 25 stars detected in all epochs and brighter than $K \sim 13$ mag.

${ }^{\mathrm{f}}$ Half of the images were taken using a narrow band $\mathrm{CO}$ filter, with the shorter exposure time, and the other half using a narrowband $K_{\text {cont }}$ filter, with the longer exposure time.

2003 and 2007 (see Gezari et al. 2002 and Ghez et al. 2003 for details of the 2000-2002 measurements).

\section{DATA EXTRACTION}

\subsection{Image Analysis and Astrometry}

The individual speckle and adaptive optics data frames are processed in two steps to create a final average image for each of the 34 imaging observing runs. First, each frame is sky-subtracted, flat-fielded, bad-pixel-corrected, corrected for distortion effects, and, in the case of the speckle data, resampled by a factor of 2 ; the distortion correction applied to the NIRC2/LGSAO data is from the NIRC2 preship review results, ${ }^{8}$ and those applied to the speckle data sets are the combined transformations given in Ghez et al. (1998) and Lu et al. (2008). The frames are then registered on the basis of the position of IRS 16C, for the speckle images, and a cross-correlation of the entire image, for the LGSAO image, and

\footnotetext{
${ }^{8}$ See http://www2.keck.hawaii.edu/inst/nirc2/preship_testing.pdf.
}

combined. For the adaptive optics data sets, the frames whose PSF has a FWHM $<1.25 \times \mathrm{FWHM}_{\min }$, where $\mathrm{FWHM}_{\text {min }}$ is the minimum observed FWHM for each epoch and which typically includes $\sim 70 \%$ of the measured frames, are combined with a weighted average with weights set equal to their Strehl ratios. To increase the $\mathrm{S} / \mathrm{N}$ of the 2005 June data set, the data taken through the two narrowband filters are averaged together. For the speckle data set, only the best $\sim 2000$ frames from each observing run are combined, using a weighted "shift-and-add" technique described by Hornstein (2007). The selected frames from each observing run (speckle and LGSAO) are also divided into three independent subsets from which three subset images are created in a similar manner to the average images; these subset images are used to assess photometric and astrometric measurement uncertainties. Figure 1 shows examples of the final average LGSAO and speckle images. While all the images sets have point-spread function (PSF) cores that are nearly diffraction-limited $\left(\theta \sim 0.06^{\prime \prime}\right.$ vs. $\left.\theta_{\text {diff.lim }}=0.05^{\prime \prime}\right)$, the LGSAO images have much higher image quality than the speckle images,

TABLE 2

Summary of New Keck Spectroscopic Observations

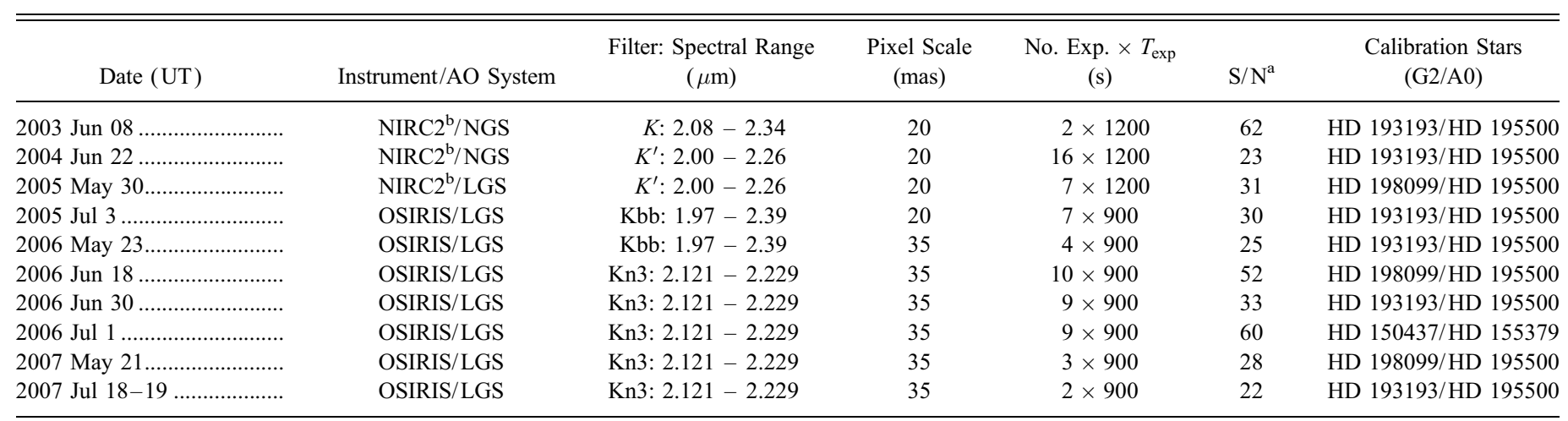

\footnotetext{
a The SNR is per spectral pixel and is calculated between 2.13 and $2.145 \mu \mathrm{m}$. The width of a spectral pixel is roughly 2.5 and $2.53 \AA$ for OSIRIS and NIRC2, respectively.
}

${ }^{\mathrm{b}}$ For the NIRC2 data sets, the slit P.A. was $259.4^{\circ}(2003), 333.76^{\circ}$ (2004), and $355.9^{\circ}$ (2005). 


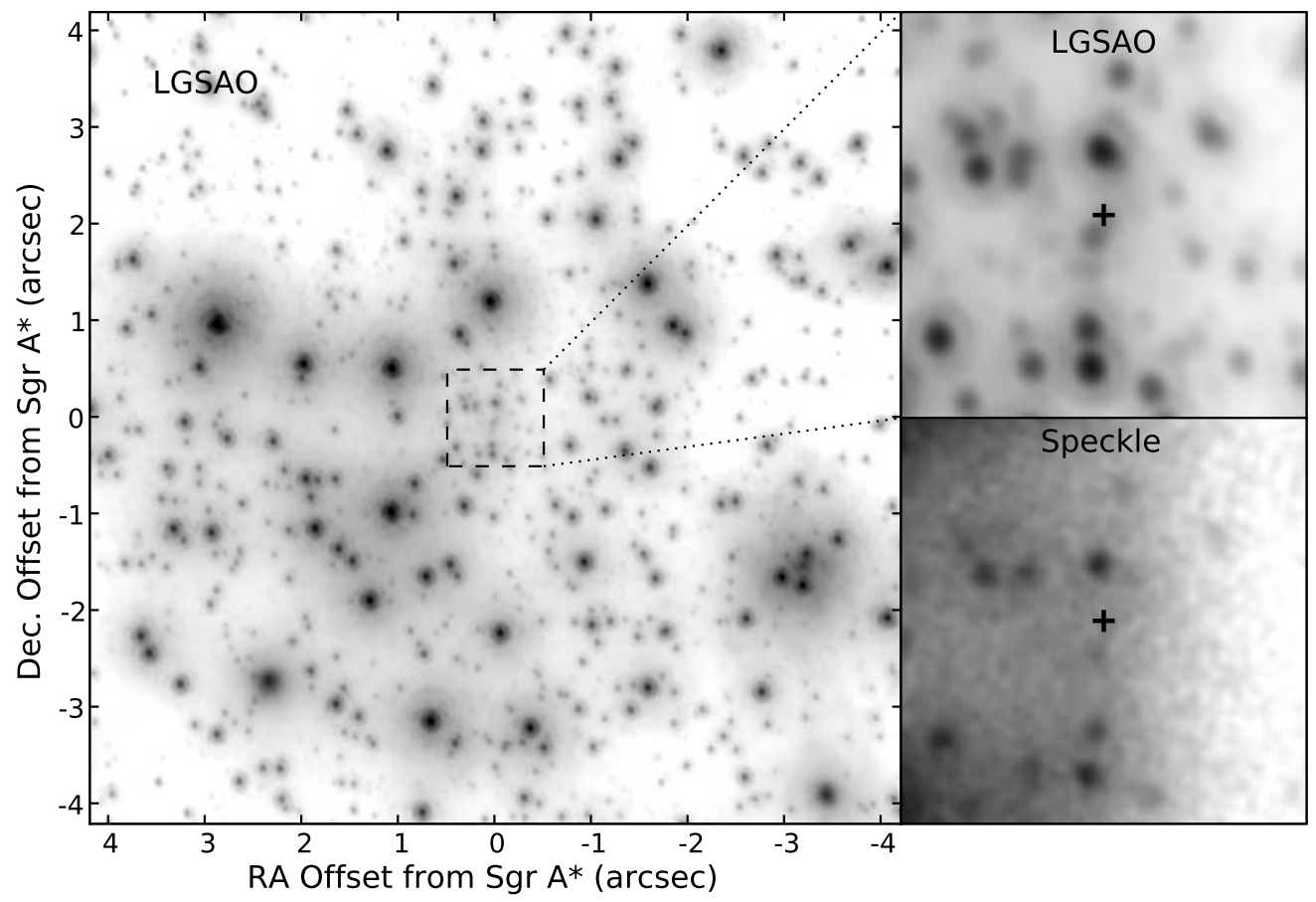

FIG. 1.-Comparison of raw images obtained with LGSAO and speckle imaging with the Keck $10 \mathrm{~m}$ telescopes. The large-scale image is an LGSAO image obtained in 2005. The inset LGSAO image (top right) and speckle image (bottom right), also obtained in 2005 , are centered on the black hole, Sgr A* (marked with a cross), with a field of view of $1.0^{\prime \prime} \times 1.0^{\prime \prime}$. The image quality, depth, and astrometric precision have all been greatly improved with the advent of LGSAO.

with median Strehl ratios of $\sim 0.3$ and 0.07 , for the LGSAO and speckle images, respectively.

Point sources are identified and characterized in each of the images using the PSF-fitting program StarFinder (Diolaiti et al. 2000) on both the average images and the subset images. StarFinder iteratively generates a PSF based on user selected point sources ${ }^{9}$ in the image and identifies additional sources in the image by cross-correlating the resulting PSF with the image. The initial source list for each image is composed only of sources detected in the average images with correlation values above 0.8 and in all three subset images with correlation values above 0.6 . Eleven bright $(K<14 \mathrm{mag})$, nonvariable sources establish the photometric zero points for each list based on measurements made by Rafelski et al. (2007; IRS 16C, IRS 16SW-E, S2-17, S1-23, S1-3, S1-4, S2-22, S2-5, S1-68, S0-13, S1-25). As shown in Figure 2, the deep LGSAO images $\left(K_{\lim } \sim 19 \mathrm{mag}\right)$ are $3 \mathrm{mag}$ more sensitive than the speckle images $\left(K_{\lim } \sim 16 \mathrm{mag}\right)$, which results in roughly 3 times more sources being detected in the LGSAO images than the speckle images over a comparable region. Because of the higher S/N, as shown in Figure 3, the centroiding uncertainties $\left(\delta X^{\prime}, \delta Y^{\prime}\right)$, which are estimated from the rms error of the measurements in the three subset images, are a factor of 6 more precise for the deep LGSAO data sets ( 0.17 mas) than the speckle data sets $(1.1 \mathrm{mas})$, for bright stars $(K<13 \mathrm{mag})$; the plateau observed in the relative centroiding uncertainties for the brighter stars $(K<13)$ in the LGSAO images is likely caused by the combined effects of differential tip-tilt jitter and residual optical distortions across the field of view.

The sources identified each night are matched across multiple epochs and their positions are transformed to a common coordinate

\footnotetext{
${ }^{9}$ In this analysis, the stars that are input into the PSF construction are IRS 16C, $16 \mathrm{NW}$, and S2-17 for the speckle images and IRS 16C, $16 \mathrm{NW}, 16 \mathrm{NE}, 16 \mathrm{SW}, 33 \mathrm{E}$, $33 \mathrm{~W}, 7,29 \mathrm{~N}$, and GEN $+2.33+4.60$ for the LGSAO images.
}

system that will be referred to as the cluster reference frame. As detailed in Appendix A, the transformation for each epoch is derived by minimizing the net displacement of a set of "coordinate reference" stars, allowing for proper motions, relative to their positions in a common reference image, which, in this case, is the 2004 July LGSAO image. This procedure attempts to ensure that
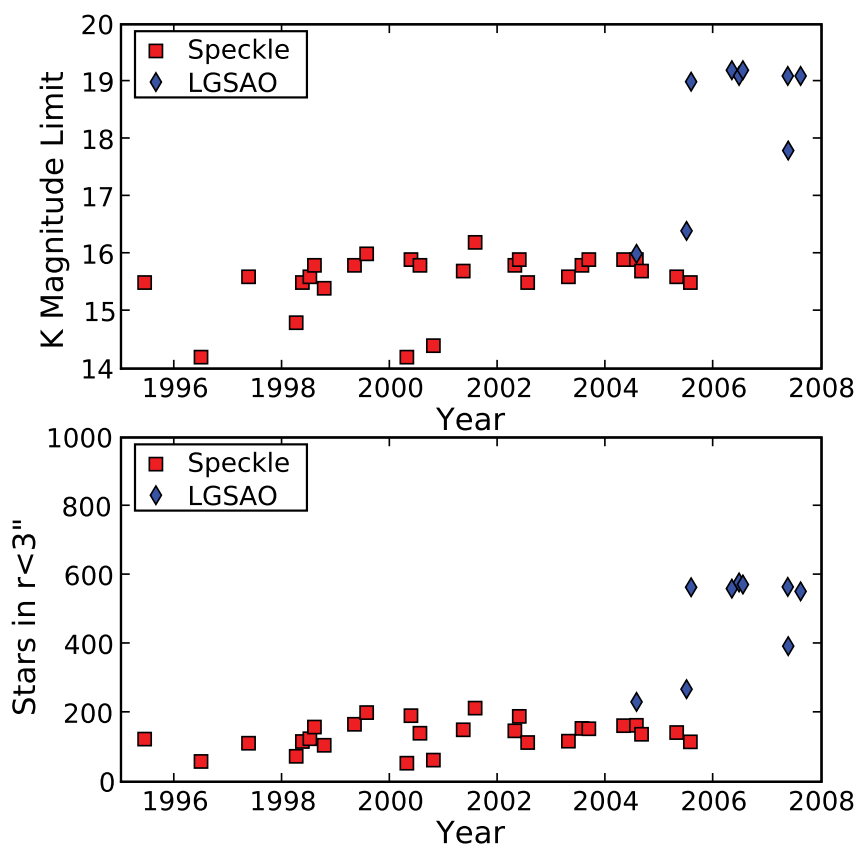

FIG. 2.-Comparison of the sensitivity of the average images from each epoch. The recent LGSAO images, with significantly longer on-sky integration times $\left(t_{\text {tot }} \sim 50\right.$ vs. 3 minutes) and much higher Strehl ratios, are 3 mag more sensitive than any of the speckle images. 


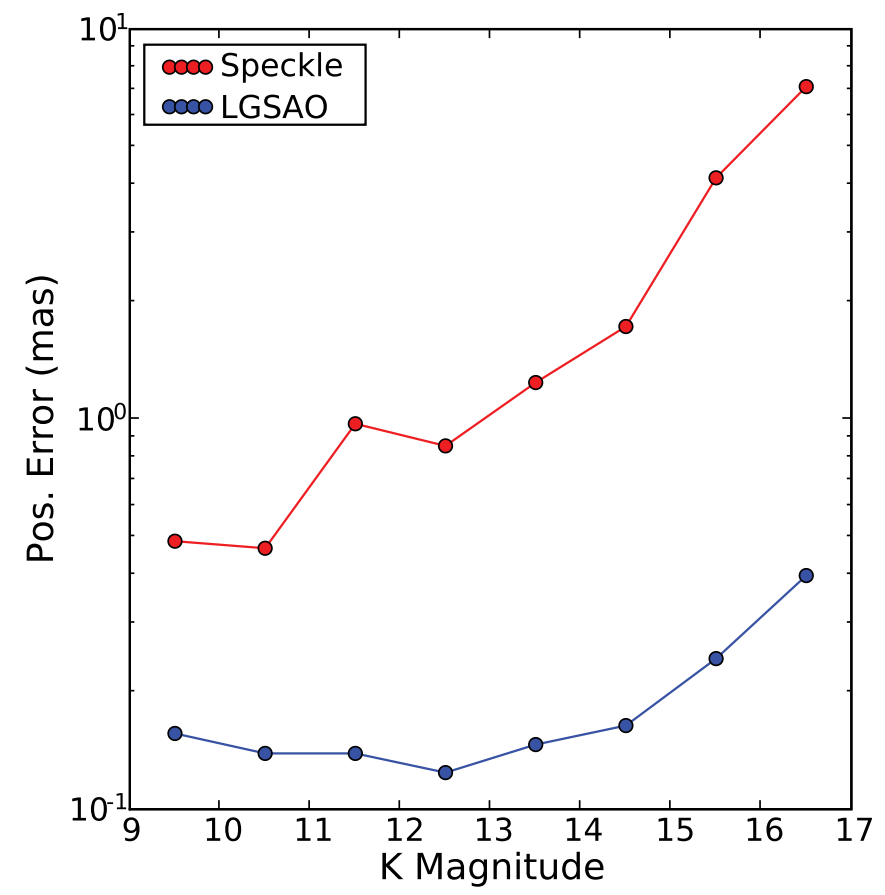

FIG. 3.-Comparison of the centroid uncertainties as a function of brightness. Because the very brightest stars $(K \sim 9)$ are saturated in their cores in the LGSAO images, there is a slight rise in their centroid uncertainties compared to somewhat fainter sources. Overall, however, for bright sources $(K<13)$, the long-exposure LGSAO images achieve a centroiding uncertainty of just 0.17 mas, a factor of $\sim 6$ better than the earlier work done with speckle imaging.

in the cluster reference frame the coordinate reference stars are at rest (i.e., no net translation, rotation, expansion, or skew). A total of $\sim 470$ and $\sim 120$ stars serve as coordinate reference stars in the LGSAO and speckle epochs, respectively. These stars are selected based on the following criteria: (1) high detection correlations $(>0.9)$, ensuring good positional accuracy, (2) location more than $0.5^{\prime \prime}$ from $\mathrm{Sgr} \mathrm{A}^{*}$ to avoid sources with measurable nonlinear motions (i.e., accelerations in the plane of the sky $>\sim 8 \mathrm{~km} \mathrm{~s}^{-1} \mathrm{yr}^{-1}$ ), (3) low velocities $\left(<15 \mathrm{mas} \mathrm{yr}^{-1}\right.$, or equivalently $\left.\sim 600 \mathrm{~km} \mathrm{~s}^{-1}\right)$, which eliminates possible coordinate reference sources that have been mismatched across epochs, and (4) lack of spectroscopic identification as a young star from Rafelski et al. (2007) to eliminate the known net rotation of the young stars in the cluster reference frame. Positional uncertainties from this transformation process, which are characterized by a half sample bootstrap applied to the coordinate reference stars, are a factor of $\sim 1.5$ (speckle) to 6 (LGSAO) smaller than the centroiding uncertainties and grow by less than a factor of 2 between the center of the field of view (minimum) and a radius of $3^{\prime \prime}$.

An additional source of positional error originates from residual optical distortion in NIRC2. While the residual distortion in NIRC2 is small, the extremely precise centroid measurements in the deep LGSAO images make it a significant effect. The presence of such a systematic error is established by examining the distribution of positional residuals, normalized by measurement (centroiding plus alignment) uncertainties, to the linear propermotion fits for the coordinate reference stars. The speckle data sets do not show large, measurable biases; the speckle measurements, on average, are only $1 \sigma$ off from the linear proper-motion fit. In contrast, the much more precise deep-LGSAO astrometric measurements are, on average, $5 \sigma \mathrm{off}$ from these fits. As described in Appendix B, we account for this effect at two stages of our analysis. First, 0.88 mas is added in quadrature to the positional uncertainties of the coordinate reference stars to account for systematic errors in the coordinate transformations. Second, a local correction, in the coordinate reference frame, is derived and applied to the positions of the short period stars that were made with LGSAO setups that differ from that of the reference image. This procedure ensures that residuals from both linear propermotion fits to the coordinate reference stars (see Appendices A and $\mathrm{B}$ ) and from orbit fits to S0-2 (see $\S 4$ ) are consistent with a normal distribution.

Source confusion can introduce positional biases that can be comparable to and, at certain times, larger than the statistical errors caused by background or detector noise. This occurs when two stars are sufficiently close to each other that only one source, rather than two, is identified in our analysis with a brightness that includes flux from both sources and a position that corresponds roughly to the photocenter of the two stars. We divide the problem of handling source confusion in our data set into the following two cases: (1) the impact of unresolved, underlying stars that are known sources, because they were sufficiently well separated at other times, and bright enough, to be independently detected, and (2) the impact of unresolved, underlying stars that are not identified by this study at another time. Because the sources are moving so rapidly, instances of the former case are easily identified and are typically blended for $1 \mathrm{yr}$. An underlying source that is comparably bright to the source of interest can have a significant impact on the astrometry; to quantify this effect, we examine the idealized, noise-free case of a perfectly known PSF by using our empirical PSFs to generate idealized binary stars and running StarFinder on these simulated images, inputting the known PSF. In this case, the astrometric bias is zero once the two components are detected. As Figure 4 shows, when the sources are blended, the resulting astrometric biases can be easily as large as 10 mas, which is much larger than our centroiding uncertainties. Such a large astrometric bias occurs when the underlying source is at least half as bright as the primary source and has a projected, although unresolved, separation of $\sim 40$ mas. We conservatively choose to eliminate all astrometric measurements that are known to be the blend of two sources from the orbital analysis; specifically, if the predicted positions of two known sources are separated by less than 60 mas and only one of them is detected, then that measurement is removed from our analysis. For S0-2 $(K=14.2 \mathrm{mag})$, the eliminated data points are those made in 1998 , due to confusion with S0-19 $(K=15.6 \mathrm{mag})$, in 2002, due to overlap with $\operatorname{Sgr} \mathrm{A}^{*}-\mathrm{IR}\left(K_{\text {median }}=16.4 \mathrm{mag}\right.$, but can be as bright as 14 mag; see Do et al. 2008), and in 2007 May, due to superposition with S0-20 $(K=15.9)$. The impact of these overlapping sources, in the first two cases, can be seen in the photometric measurements (see Fig. 5).

Source confusion from unknown sources is a smaller effect than that from known sources, since the unknown stars, in general, are fainter than the known sources. Given the long time baseline of the speckle imaging experiment, knowledge of sources in this region is most likely complete down to $K=16.0 \mathrm{mag}$. While sources as faint as $K=19$ mag have been detected in this region with LGSAO, crowding and the short time baseline of these deeper observations limit the census of these sources. Therefore, source confusion from unknown sources can give rise to astrometric biases for S0-2 as large as 3 mas (from a $K=16$ mag source), but are typically significantly smaller, since underlying sources will generally be fainter than $K=16 \mathrm{mag}$. To characterize the expected astrometric bias from the undetected source distribution, a Monte Carlo simulation was performed by generating multiple images with all known stars plus a random stellar distribution that, in total, follows the $K$ luminosity function and radial profile 


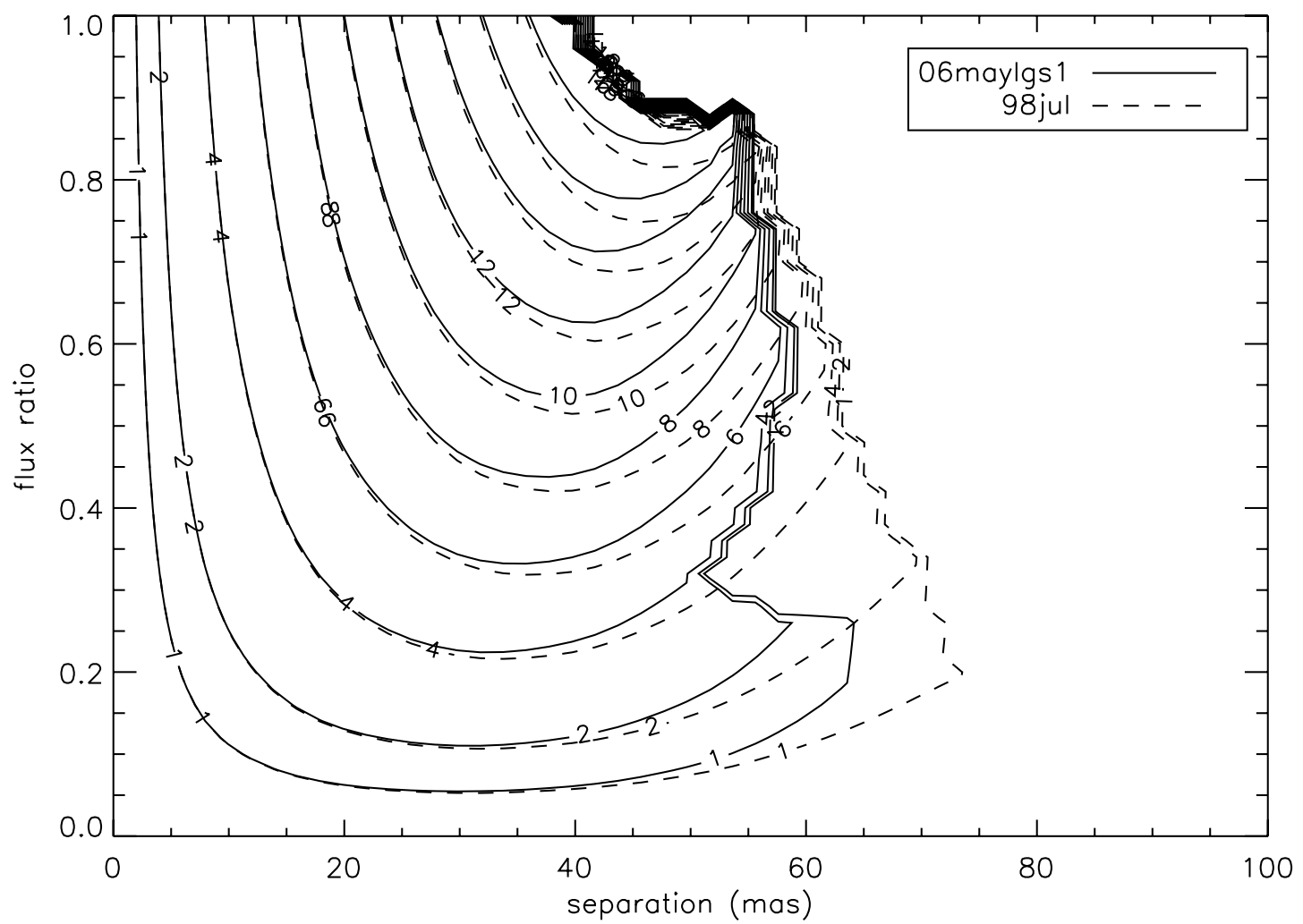

FIG. 4.- Astrometric bias introduced by an unresolved source in the case of a binary star generated and analyzed with a known PSF. Two cases are shown: PSF from LGSAO image in 2006 May (solid line) and PSF from speckle image in 1998 July (dashed line). The contour lines show the amount of bias (in mas) introduced by an underlying source of the indicated flux ratio and separation. Once the neighboring source is detected, which happens at separations of $\sim 60$ mas, the astrometric bias drops to zero in this idealized case. For 1: 1 binaries, pairs with smaller separations can be resolved. This figure shows that biases well above the positional uncertainties ( $\sim 1$ mas) can occur due to underlying sources.
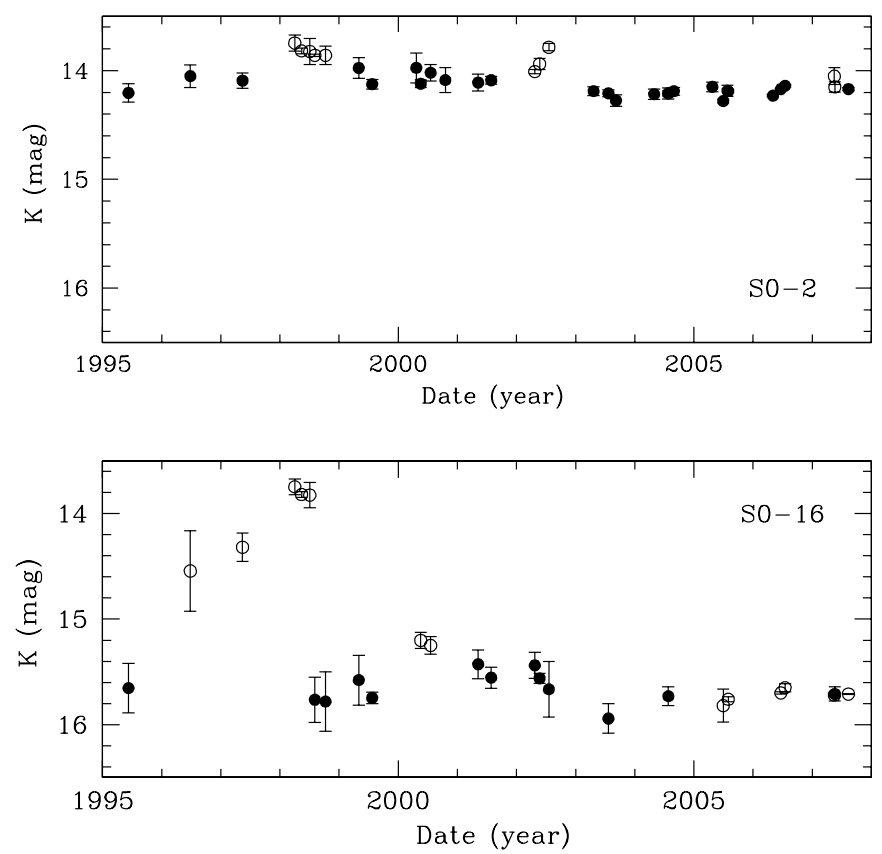

Fig. 5.-Photometric measurements vs. time for S0-2 (top) and S0-16 (botttom). Measurements that were made when these sources coincided with another known source are plotted as open points and excluded from the model-fitting procedure. S0-16 is more affected by underlying sources, because it is fainter. Even without a priori knowledge of the underlying sources, their effect is clearly visible in photometric measurements made in 1998 and 2002, for S0-2, and 1996-1999 and 2000, for S0-16. from Schödel et al. (2007). By running these simulated images through our data analysis procedure, we estimate that the astrometric error from unknown sources for $\mathrm{S} 0-2$ is, on average, 0.5 and 1.2 mas for the LGSAO and speckle images, respectively, and that it scales roughly with the photometric bias and galactocentric distance. However, it should be noted that the exact value of this bias is model dependent. While the photometric bias may be detected in the speckle data toward closest approach (see Fig. 5), the estimated astrometric biases are smaller than other sources of positional uncertainty already included for the majority of the $\mathrm{S} 0-2$ data points. We therefore do not incorporate them into the reported positional uncertainties. Confusion with unknown sources gives rise to larger astrometric biases for S0-16, S0-19, and S0-20, since these sources are fainter than S0-2. Given the velocity dispersion in this region and the angular resolution of the data sets, the expected timescale associated with biases from source confusion is $\sim 1-2$ yr.

As a final step, the relative astrometric positions are placed in an absolute coordinate reference frame using the positions of seven $\mathrm{SiO}$ masers (Reid et al. 2003, 2007). Infrared observations of these masers with the Keck II LGSAO/NIRC2 system between 2005 and 2007 were obtained with the same camera (i.e., plate scale) used for the precision astrometry measurements described above, but with a nine-position box pattern and a $6^{\prime \prime}$ dither offset to create a $22^{\prime \prime} \times 22^{\prime \prime}$ mosaic of these masers (see Appendix C for details). A comparison of the maser positions measured in this infrared mosaic to the predicted radio positions at this epoch from Reid et al. (2003) establishes that the mosaic has an average pixel scale of $9.963 \pm 0.005$ mas pixel $^{-1}$ and a position angle of north with respect to the NIRC2 columns of $0.13^{\circ} \pm 0.02^{\circ}$. This same analysis localizes the radio position of Sgr $\mathrm{A}^{*}$ in the infrared 
mosaic to within 5 mas in the east-west and north-south directions. By aligning the infrared stars detected in both the larger infrared mosaic and the precision astrometry image taken during the same observing run, we have the necessary coordinate transformations to convert our relative astrometric position measurements into an absolute reference frame. For the orbit analysis described in $\S 4$, the uncertainties in this transformation are applied only after model orbits have been fit to the relative astrometry and are a negligible source of uncertainty in the final mass and $R_{0}$ estimates.

\subsection{Spectral Analysis and Radial Velocities}

In the analysis of the spectral data, we accomplish the initial basic data processing steps using standard IRAF procedures, for NIRC2 and NIRSPEC, and a facility IDL data extraction pipeline for OSIRIS. Specifically, each data set is first (1) flat-fielded, (2) dark-subtracted, (3) bad-pixel- and cosmic-ray-corrected, (4) spatially dewarped, and (5) wavelength calibrated. Wavelength calibration is performed by identifying $\mathrm{OH}$ emission lines from sky spectra and fitting a low-order polynomial function to the location of the lines. For the NIRSPEC spectra, neon emission lines from arc lamps provide the wavelength calibration. The accuracy of the wavelength calibration is $\sim 9 \mathrm{~km} \mathrm{~s}^{-1}$ or less for NIRC2 and OSIRIS as measured by the dispersion of the residuals to the fit. Next, the one-dimensional stellar spectra are extracted using a spatial window that covers $\sim 0.1^{\prime \prime}$ for the two-dimensional spectral data sets from NIRC2 and NIRSPEC. For the three-dimensional spectral data set from OSIRIS, an extraction box $0.14^{\prime \prime} \times 0.14^{\prime \prime}$ was used. To correct for atmospheric telluric absorption features, each spectrum is divided by the spectrum of an A-type star. Prior to this step, the A-type star's strong intrinsic $\mathrm{Br} \gamma$ feature is removed. In the case of the NIRC2 and OSIRIS observations, this correction is done with observations of a G2 V star, which is divided by a model solar spectrum. The $\mathrm{Br} \gamma$-corrected region in the $\mathrm{G}$ star is then substituted into the same region of the A star (Hanson et al. 1996). In the case of the NIRSPEC observations, the A-type star's Br $\gamma$ feature is corrected with a model spectrum of $\mathrm{Vega}^{10}$ rebinned to the resolution of the A-type star's spectrum and convolved with a Gaussian to match the spectral resolution of the observations. The resulting stellar spectra are corrected for all telluric absorption features; however, they are still contaminated by background emission due to the gas around the Galactic center. The local background is estimated and removed by subtracting spectra extracted from regions that are $\sim 0.1^{\prime \prime}$ away. Finally, all the spectra within each night of observation are combined in an average, weighted by the $\mathrm{S} / \mathrm{N}$.

$\mathrm{RV}$ estimates are determined for each spectrum on the basis of the location of the $\mathrm{Br} \gamma$ line. While a few of our spectra with broader spectral coverage also show a weaker He I triplet at $2.116 \mu \mathrm{m}$, we do not incorporate measurements from this line, as it is a blend of transitions that can bias the resulting radial velocities (see Fig. 6). A Gaussian model is fit to each of the $\mathrm{Br} \gamma$ line profiles and the wavelength of the best-fit peak, is compared to the rest wavelength of $\lambda_{\text {vacuum }}=2.1661 \mu \mathrm{m}$ to derive an observed RV. To obtain radial velocities in the local standard of rest (LSR) reference frame, each observed RV is corrected for the Earth's rotation, its motion around the Sun, and the Sun's peculiar motion with respect to the LSR $\left(U=10 \mathrm{~km} \mathrm{~s}^{-1}\right.$, radially inwards; Dehnen \& Binney 1998). Since the LSR is defined as the velocity of an object in circular orbit at the radius of the Sun, the Sun's peculiar motion with respect to the average velocity of stars in its vicinity should give the Sun's motion toward the center of the Galaxy.

\footnotetext{
${ }^{10}$ Model taken from the 1993 Kurucz Stellar Atmospheres Atlas (http://www .stsci.edu/hst/observatory/cdbs/k93models.html).
}

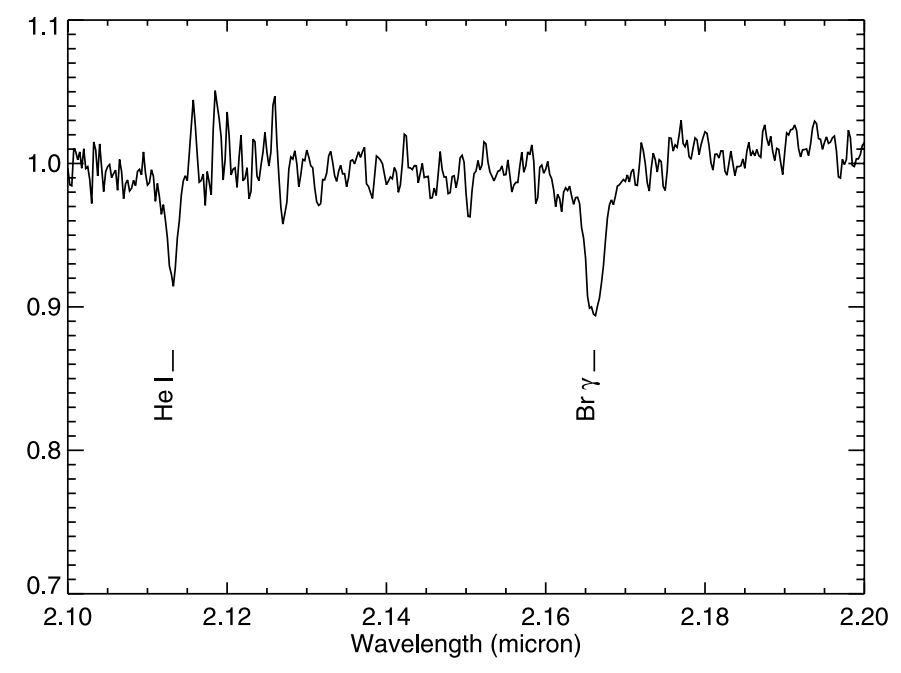

FIG. 6. - Weighted average of all S0-2 spectra obtained with the W. M. Keck II telescope. Since only some of the data sets contain the shorter wavelengths, the $\mathrm{S} / \mathrm{N}$ is lower at wavelengths shortward of $2.13 \mu \mathrm{m}$. While $\mathrm{Br} \gamma$ and $\mathrm{He}$ I lines are clearly detected, only the $\mathrm{Br} \gamma$ line, which is stronger and not the blend of multiple lines, is used to measure the radial velocity of S0-2 as a function of time.

The uncertainties in the final radial velocities are obtained from the rms of the fits to the line profile measurements from at least three independent subsets of the original data set. Figure 7 shows how S0-2's Br $\gamma$ line has shifted over time and how the measurement of this line has improved by a factor of 5 with improved instrumentation. For the deep LGSAO spectroscopic observations, the RV uncertainties for S0-2 are typically $\sim 20-25 \mathrm{~km} \mathrm{~s}^{-1}$.

\section{ORBITAL ANALYSIS AND RESULTS}

\subsection{Point Mass-Only Analysis}

To derive the black hole's properties, we assume that the stars are responding to the gravitational potential of a point mass. In this analysis, the seven properties of the central black hole that are fitted are its mass $(M)$, distance $\left(R_{0}\right)$, location on the plane of the sky $\left(X_{0}, Y_{0}\right)$ and motion $\left(V_{x}, V_{y}, V_{z}\right)$. In addition to these common free parameters, there are the following six additional free parameters for each star: period $(P)$, eccentricity $(e)$, time of periapse passage $\left(T_{0}\right)$, inclination $(i)$, position angle of the ascending node $(\Omega)$, and the longitude of periapse $(\omega)$. Using a conjugate gradient $\chi^{2}$ minimization routine that simultaneously fits the astrometric and RV measurements, we fit this model to measurements that are given in Tables 3 and 4, which includes 27 epochs of astrometric measurements and 11 epochs of RV measurements, as well as five additional epochs of radial velocity measurements reported in the literature (Eisenhauer et al. 2003, 2005). This excludes all the astrometric measurements of S0-2 that are confused with another known source (see $\S 3.1$ ). While the 2002 astrometric data are eliminated due to confusion with $\mathrm{Sgr} \mathrm{A}^{*}$, the $2002 \mathrm{RV}$ points are not, since $\mathrm{Sgr} \mathrm{A}^{*}$ is featureless and therefore does not bias the measurement of RV from S0-2's Br $\gamma$ absorption line. In total, there are 38 astrometric data points and 5 RV measurements. All values reported for each parameter are the best-fit values obtained from minimizing the total $\chi_{2}^{2}$, which is the sum of the $\chi^{2}$ from each data type (i.e., $\chi_{\text {tot }}^{2}=\chi_{\text {ast }}^{2}+\chi_{\mathrm{RV}}^{2}$ ).

The uncertainties on the fitted parameters are estimated using a Monte Carlo simulation, which is a robust approach when performing a fit with many correlated parameters. We created $10^{5}$ artificial data sets $\left(N_{\text {sim }}\right)$ containing as many points as the observed data set (astrometry and radial velocities), in which each point is 

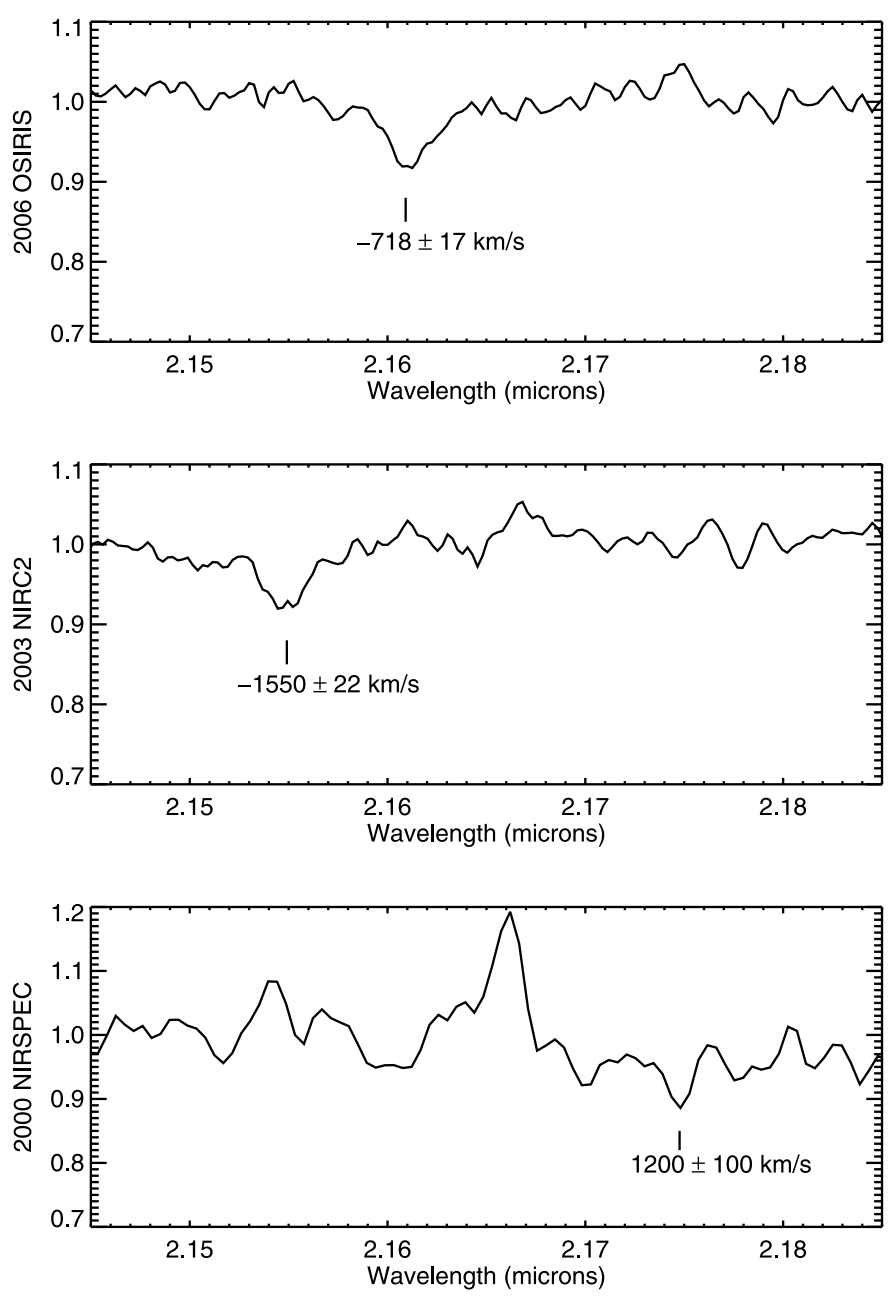

FIG. 7.-Measurements of S0-2's Br $\gamma$ line. These three measurements show that, over time, S0-2's radial velocity has changed by more than $2600 \mathrm{~km} \mathrm{~s}^{-1}$. In order to improve the line detection in the low S/N NIRSPEC observation, the emission from the local gas was not removed, which leaves a large $\mathrm{Br} \gamma$ emission feature centered at small radial velocities compared to that of the star at this time. With improvements in the adaptive optics system and instrumentation (from NIRSPEC/NGSAO [bottom], to NIRC2/NGSAO [middle], and finally to OSIRIS/ LGSAO [top]), the precision with which the $\mathrm{Br} \gamma$ absorption line can be measured in S0-2 has improved by a factor of 5 .

randomly drawn from a Gaussian distribution centered on the actual measurement and whose $1 \sigma$ width is given by the associated uncertainty, and run the $\chi^{2}$ minimization routine for each realization. $N_{\text {sim }}$ was set to $10^{5}$ in order to achieve $\sim 6 \%$ accuracy in the resulting estimates of the a $99.73 \%$ confidence limits ( $3 \sigma$ equivalent for a Gaussian distribution) of the orbital parameters. Because the $\chi^{2}$ function contains many local minima, each realization of the data is fit 1000 times $\left(N_{\text {seed }}\right)$ with different seeds to find the global minimum. The resulting distribution of $10^{5}$ values of the fitted parameters from the Monte Carlo simulation, once normalized, is the joint probability distribution function of the orbital parameters $[\operatorname{PDF}(\boldsymbol{O})$, where $\boldsymbol{O}$ is a vector containing all the orbital parameters, $O_{i}$ ]. For each orbital parameter, $\operatorname{PDF}(\boldsymbol{O})$ is marginalized against all other orbital parameters to generate a $\operatorname{PDF}\left(O_{i}\right)$. The confidence limits for each parameter are obtained by integrating each $\operatorname{PDF}\left(O_{i}\right)$ from its peak ${ }^{11}$ outwards to a probability of $68 \%$.

\footnotetext{
11 While the best values from minimizing $\chi^{2}$ can differ slightly (but well within the uncertainties) from the peak of the $\operatorname{PDF}\left(O_{i}\right)$ values, this has negligible impact on the reported uncertainties.
}

Compared to all other stars at the center of the Milky Way, S0-2 dominates our knowledge of the central black hole's properties. Two facts contribute to this effect. Most importantly, it has the shortest known orbital period ( $P=15$ yr; Schödel et al. 2002, 2003; Ghez et al. 2003, 2005a). Furthermore, among the known shortperiod stars, it is the brightest star and therefore the least affected by stellar confusion (see Fig. 1). Several other stars, in principle, also offer constraints on the black hole's properties. In particular, S0-16 is the next most kinematically important star, as it is the only other star that yields an independent solution for the black hole's properties. However, independent solutions for the black hole's position from fits to S0-2 and S0-16 measurements differ by more than $5 \sigma$ (see Fig. 8). While S0-16's measurements in 2000 have already been omitted due to overlap with the position of Sgr A*, three independent lines of reasoning lead us to believe that some of S0-16's remaining astrometric measurements must be significantly biased by radiation from unrecognized, underlying stars. First, as shown in Figure 4, unknown sources can introduce astrometric biases as large as 9 mas for $\mathrm{S} 0-16(K=15)$, in contrast with only 3 mas for S0-2 $(K=14)$, because it is only 1 mag above the completeness limit for detection in the speckle data set $(K \sim 16$ mag; see $\S 3.1)$. Second, a comparison of the solution for the position of the black hole $\left(X_{0}, Y_{0}\right)$ based on both the astrometric and radial velocity measurements to that based on astrometry alone (fixing the distance, which cannot be solved for without radial velocities) yields a consistent position from modeling the two cases for S0-2, but produces different results for the two cases from modeling S0-16's measurements, with the inferred $X_{0}$ and $Y_{0}$ from astrometry alone shifting further away from that obtained from modeling S0-2's orbit prediction and thereby increasing the discrepancy to $10 \sigma$. Third and finally, while the position of the dynamical center from S0-2's orbit is statistically consistent with $\mathrm{Sgr} \mathrm{A}^{*}$-radio/IR, which is the emissive source associated with the central black hole (e.g., Melia \& Falcke 2001; Genzel et al. 2003a; Ghez et al. 2004; 2005b; Hornstein et al. 2007), the solution from S0-16 is not (see Fig. 8); this difference cannot be explained by allowing the black hole to move with time or by introducing an extended mass distribution. We therefore restrict our remaining analysis to $\mathrm{S} 0-2$.

As shown in Figures 9 and 10, the astrometric and radial velocity measurements for S0-2 are well fit by a simple Keplerian model. For a 13-parameter model (right-hand panels), the best fit to the data produces a total $\chi^{2}$ of 54.8 for 57 degrees of freedom (dof) and a $\chi^{2} /$ dof of 0.961 . From the Monte Carlo simulation, we derive probability distributions for the central black hole's properties, which are shown in Figure 11 and characterized in Table 5. These distributions give a best fit for the central black hole's mass of $M_{\mathrm{bh}}=4.1 \pm 0.6 \times 10^{6} M_{\odot}$ and distance of $R_{0}=$ $8.0 \pm 0.6 \mathrm{kpc}$ (all quoted uncertainties are $68 \%$ confidence values). The position of the black hole is confined to within \pm 1 mas ( $\sim 100$ Schwarzschild radii). As can be seen in Figure 11, the inferred black hole's mass is highly correlated with its distance. Estimates from orbital modeling are expected to have a power law relationship of the form mass $\propto M_{\odot}$ distance ${ }^{\alpha}$ with $\alpha$ between 1 and 3. For the case of astrometric data only, $\alpha$ should be 3 and, for the case of radial velocity data only, $\alpha$ is expected to be 1 . Currently, the relationship is $M=\left(4.1 \pm 0.1 \times 10^{6} M_{\odot}\right)\left(R_{0} / 8.0 \mathrm{kpc}\right)^{1.8}$, which suggests that the astrometric and radial velocity data sets are having roughly equal affect in the model fits for mass. ${ }^{12}$

A fit that includes the biased astrometric data points significantly alters the best-fit solution for S0-2. Including both the

\footnotetext{
12 The uncertainty in the mass scaling relationship is obtained for the case in which $R_{0}$ is fixed to $8.0 \mathrm{kpc}$ and therefore does not include the uncertainty in $R_{0}$.
} 
TABLE 3

Summary of Keck Astrometric and Photometric Measurements

\begin{tabular}{|c|c|c|c|}
\hline UT Date & $\begin{array}{c}K_{\mathrm{obs}} \\
(\mathrm{mag})\end{array}$ & $\begin{array}{c}X \\
{\left(\mathrm{mas}^{\mathrm{a}}{ }^{\mathrm{a}}\right.}^{2}\end{array}$ & $\begin{array}{c}Y \\
{\left(\mathrm{mas}^{\mathrm{a}}{ }^{\mathrm{a}}\right.}\end{array}$ \\
\hline $1995.439 \ldots$ & $14.21 \pm 0.09$ & $42.6 \pm 1.0$ & $164.10 \pm 0.98$ \\
\hline $1996.485 \ldots \ldots$ & $14.05 \pm 0.10$ & $53.0 \pm 9.5$ & $155.4 \pm 9.5$ \\
\hline 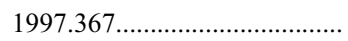 & $14.09 \pm 0.07$ & $56.5 \pm 1.7$ & $137.0 \pm 1.7$ \\
\hline 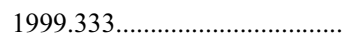 & $13.98 \pm 0.10$ & $66.6 \pm 3.1$ & $91.5 \pm 3.1$ \\
\hline $1999.559 \ldots \ldots \ldots \ldots$ & $14.12 \pm 0.04$ & $67.4 \pm 1.4$ & $88.3 \pm 1.4$ \\
\hline $2000.305 \ldots \ldots$ & $13.98 \pm 0.14$ & $64.3 \pm 3.0$ & $65.8 \pm 3.1$ \\
\hline $2000.381 \ldots \ldots \ldots$ & $14.12 \pm 0.04$ & $66.7 \pm 1.1$ & $63.0 \pm 1.1$ \\
\hline $2000.548 \ldots \ldots \ldots \ldots \ldots \ldots \ldots \ldots \ldots \ldots$ & $14.02 \pm 0.08$ & $64.84 \pm 0.78$ & $57.94 \pm 0.80$ \\
\hline $2000.797 \ldots \ldots$. & $14.09 \pm 0.11$ & $65.4 \pm 4.8$ & $46.8 \pm 4.9$ \\
\hline $2001.351 \ldots \ldots \ldots \ldots$ & $14.11 \pm 0.08$ & $56.7 \pm 1.6$ & $26.5 \pm 1.6$ \\
\hline $2001.572 \ldots$ & $14.09 \pm 0.04$ & $53.0 \pm 1.4$ & $14.2 \pm 1.3$ \\
\hline $2003.303 \ldots$ & $14.19 \pm 0.04$ & $-34.9 \pm 1.5$ & $69.5 \pm 1.6$ \\
\hline $2003.554 \ldots$ & $14.21 \pm 0.03$ & $-35.45 \pm 0.90$ & $81.04 \pm 0.90$ \\
\hline $2003.682 \ldots$ & $14.28 \pm 0.05$ & $-34.5 \pm 2.3$ & $87.4 \pm 2.3$ \\
\hline $2004.327 \ldots \ldots \ldots \ldots \ldots \ldots \ldots \ldots$ & $14.22 \pm 0.05$ & $-32.15 \pm 0.84$ & $113.95 \pm 0.86$ \\
\hline 2004.564 & $14.21 \pm 0.05$ & $-28.7 \pm 1.4$ & $121.3 \pm 1.5$ \\
\hline 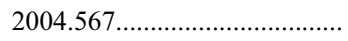 & $14.21 \pm 0.02$ & $-28.4 \pm 1.4$ & $122.9 \pm 1.4$ \\
\hline 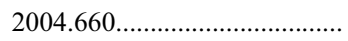 & $14.19 \pm 0.04$ & $-26.8 \pm 1.1$ & $125.5 \pm 1.1$ \\
\hline 2005.312 & $14.15 \pm 0.04$ & $-18.58 \pm 0.88$ & $142.43 \pm 0.92$ \\
\hline 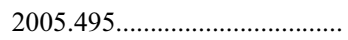 & $14.28 \pm 0.02$ & $-18.6 \pm 1.0 \pm 1.1$ & $145.3 \pm 1.0 \pm 2.5$ \\
\hline $2005.566 \ldots \ldots \ldots \ldots \ldots \ldots \ldots \ldots \ldots \ldots \ldots$ & $14.18 \pm 0.05$ & $-15.3 \pm 1.7$ & $148.9 \pm 1.8$ \\
\hline 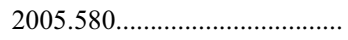 & $14.19 \pm 0.01$ & $-16.9 \pm 0.23 \pm 1.0$ & $146.8 \pm 0.23 \pm 1.5$ \\
\hline 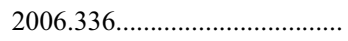 & $14.23 \pm 0.01$ & $-7.97 \pm 0.13 \pm 0.77$ & $159.82 \pm 0.13 \pm 0.66$ \\
\hline $2006.470 \ldots \ldots \ldots \ldots \ldots \ldots \ldots \ldots \ldots \ldots \ldots$ & $14.17 \pm 0.01$ & $-6.01 \pm 0.14 \pm 0.77$ & $161.57 \pm 0.14 \pm 0.66$ \\
\hline 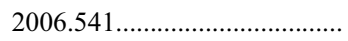 & $14.14 \pm 0.01$ & $-4.89 \pm 0.17 \pm 0.77$ & $162.26 \pm 0.17 \pm 0.66$ \\
\hline $2007.612 \ldots \ldots \ldots \ldots \ldots \ldots \ldots \ldots \ldots \ldots$ & $14.17 \pm 0.01$ & $6.88 \pm 0.20 \pm 0.77$ & $173.47 \pm 0.20 \pm 0.66$ \\
\hline
\end{tabular}

Notes. - Uncertainties from residual distortions in NIRC2 relative to the 2004 July reference image are reported separately (the second uncertainty term in the table) and should be added in quadrature to the other uncertainty terms to obtain the final positional uncertainties; since the 2006-2007 LGSAO images are all obtained with the same setup, positions from these images have correlated residual distortion uncertainties.

${ }^{\mathrm{a}} X$ and $Y$ are the relative positions in the east-west and north-south direction, with increasing values to the east and north, respectively. These values are in our absolute coordinate system (i.e., relative to Sgr A*-radio; see Appendix C), but the uncertainties do not include the uncertainties in the absolute coordinate system. Measurements that are confused with other known sources are not included in this table.

1998 and 2002 data points, which correspond to confusion with S0-19 and Sgr $A^{*}-$ IR, respectively, results in a higher mass $\left(5.7 \times 10^{6} M_{\odot}\right)$, distance $(9.4 \mathrm{kpc})$, and $\chi^{2} /$ dof $(1.7)$. Including the 2002 but not the 1998 data points also produces elevated values $\left(5.2 \times 10^{6} M_{\odot}\right.$ and $\left.9.1 \mathrm{kpc}\right)$ and $\chi^{2} /$ dof $(1.1)$. This demonstrates that it is important to account for the astrometric biases introduced by unresolved sources.

Formal uncertainties in mass and distance estimates from orbital fits can be reduced by adding a priori information. In par-

TABLE 4

Summary of Keck Radial Velocity Measurements

\begin{tabular}{|c|c|c|}
\hline \multirow[b]{2}{*}{ UT DATE } & \multicolumn{2}{|c|}{ Radial Velocity $\left(\mathrm{km} \mathrm{s}^{-1}\right)$} \\
\hline & Observed & LSR \\
\hline $2000.487 .$. & $1192 \pm 100$ & $1199 \pm 100$ \\
\hline $2003.438 \ldots$ & $-1556 \pm 22$ & $-1550 \pm 22$ \\
\hline 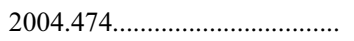 & $-1151 \pm 57$ & $-1143 \pm 57$ \\
\hline 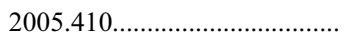 & $-945 \pm 16$ & $-926 \pm 16$ \\
\hline 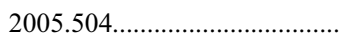 & $-853 \pm 31$ & $-850 \pm 31$ \\
\hline $2006.391 \ldots \ldots \ldots \ldots \ldots \ldots \ldots \ldots \ldots \ldots \ldots$ & $-715 \pm 21$ & $-692 \pm 21$ \\
\hline $2006.461 \ldots \ldots \ldots \ldots \ldots \ldots \ldots \ldots$ & $-728 \pm 17$ & $-718 \pm 17$ \\
\hline 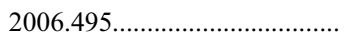 & $-699 \pm 36$ & $-695 \pm 36$ \\
\hline 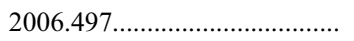 & $-717 \pm 37$ & $-713 \pm 26$ \\
\hline $2007.385 \ldots \ldots \ldots \ldots \ldots \ldots$ & $-507 \pm 50$ & $-483 \pm 50$ \\
\hline 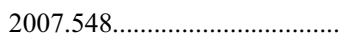 & $-502 \pm 50$ & $-506 \pm 50$ \\
\hline
\end{tabular}

ticular, it is, in principle, possible to constrain the dynamical center to be at the position of Sgr A*-IR. However, as shown in Figure 8, the six measurements of Sgr $\mathrm{A}^{*}-\mathrm{IR}$ 's position in the deep LGSAO images (2005-2007), which have the most precise astrometric measurements, have an average value that differs from the position of the black hole inferred from S0-2's orbit by 9.3 mas and a variance of 3 mas, which is a factor of 4 larger than expected from the measured positional uncertainties ( 0.7 mas). Sgr A*-IR is located where the underlying sources are expected to have the highest number density and velocity dispersion, which should induce time-variable positional biases. Sgr A*-IR's average $K$ magnitude in these deep LGSAO images is 16.4 , which is comparable to the completeness limit for sources in this region (see $\S 3.1$ ) and which is, consequently, potentially subject to large astrometric biases (see Fig. 4). We therefore suspect that the measured positions of $\mathrm{Sgr} \mathrm{A}^{*}-\mathrm{IR}$ suffer from astrometric biases from underlying sources and do not use its positions to constrain the model fits.

Another prior, which has been imposed in earlier orbital analyses of S0-2 for $R_{0}$ (Eisenhauer et al. 2003, 2005), is on the black hole's motion relative to the measurements' reference frame. Setting the three dimensional velocity to zero and fitting a 10 -parameter model $\left(\chi^{2} /\right.$ dof $=1.3$; see Figs. 9 and 10 , left-hand panels) yields uncertainties in the black hole's properties that are a factor of 2 smaller $\left(R_{0}=8.0 \pm 0.3 \mathrm{kpc}\right.$ and $M_{\mathrm{bh}}=4.4 \pm 0$. $3 \times 10^{6} M_{\odot}$ ). However, this assumption is not justified (see, e.g., Salim \& Gould 1999; Nikiforov 2008). Introducing $V_{x}$ and $V_{y}$ 


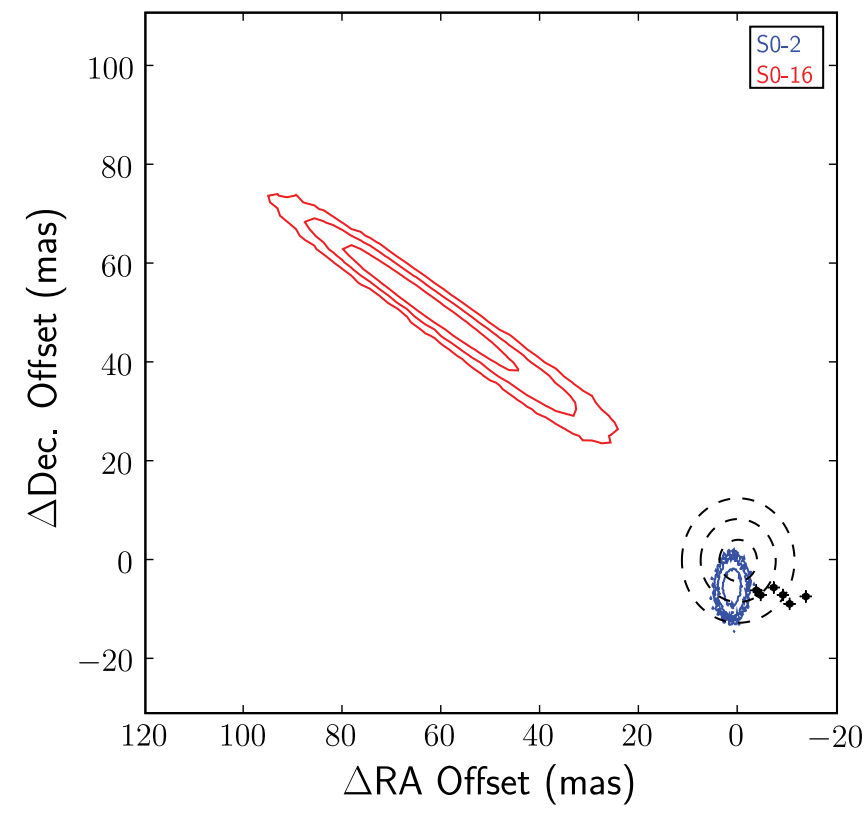

FIG. 8.-Comparison of estimates of the black hole's location. Colored contours represent the estimates of the dynamical center from model fits to kinematic measurements of S0-2 $(K=14.0$; blue $)$ and S0-16 $(K=15.0$; red $)$. Black contours show the Sgr A*-radio position. All contours are plotted at the $68 \%, 95 \%$, and $99.7 \%$ confidence levels (equivalent to 1,2 , and $3 \sigma$ for a Gaussian distribution). The solid black points are all the measurements of $\operatorname{Sgr} A^{*}-\operatorname{IR}(K \sim 16)$ in the maps used for the astrometric analysis. The discrepancy in the black hole's location from S0-16's positional measurements appear to be a consequence of biases from unrecognized, underlying stars and thus only S0-2's measurements are used to infer the properties of the central black hole. Likewise, the astrometric positions of Sgr A*-IR, which is even fainter than S0-16, also may be biased (see discussion in $\S 4.1$ ) and are therefore not used to constrain the orbital model used to fit S0-2.

(defined such that positive numbers are motions in the east and north directions, respectively) into the fit allows the dynamical center to move linearly in time in the plane of the sky with respect to the cluster reference frame. Such an apparent motion can arise from either a physical or a data analysis effect. In the case of a physical effect, the black hole could be moving with respect to the stellar cluster under the gravitational influence of a massive companion or the black hole and the cluster could be participating in a mutually opposing sloshing mode. In the case of a data analysis effect, the reference frame could be nonstationary with respect to the position of the dynamical center, which might arise if there was a systematic problem in our alignment of images. Introducing these two parameters therefore provides a way of examining possible systematic reference frame problems. Fits to a 12-parameter model ( $V_{z}$ fixed to zero) to the data have a minimum $\chi^{2} /$ dof of 0.95 , uncertainties in the black hole's properties that are larger than the 10-parameter model but smaller than the 13-parameter model $\left(R_{0}=8.4 \pm 0.4 \mathrm{kpc}\right.$ and $M_{\mathrm{bh}}=4.5 \pm 0.4 \times$ $\left.10^{6} M_{\odot}\right)$, and an estimate for the black hole's motion relative to the central stellar cluster of $V_{x}=-0.40 \pm 0.25 \mathrm{mas} \mathrm{yr}^{-1}(17 \pm$ $\left.11 \mathrm{~km} \mathrm{~s}^{-1}\right)$ and $V_{y}=0.39 \pm 0.14 \mathrm{mas} \mathrm{yr}^{-1}\left(16 \pm 6 \mathrm{~km} \mathrm{~s}^{-1}\right)$. Since these relative velocities are comparable to the constraints on the IR reference frame's motion with respect to Sgr A*-radio (i.e., an absolute reference frame in which the black hole's position is known; see Appendix C), it is important to leave $V_{x}$ and $V_{y}$ as free parameters, even for the case in which one assumes that the black hole has no intrinsic motion with respect to the cluster. Because the black hole is so often assumed to be at rest, we report the complete solution for the 12-parameter fit ( $V_{z}$ fixed to zero) in Table 5 .
As Figure 12 shows, the black hole's motion along the line of sight with respect to our assumed local standard of rest $\left(V_{z}\right)$ dominates the uncertainties in $R_{0}$ in our 13-parameter model. Priors on $V_{z}$ therefore have a significant impact on the resulting uncertainties. Unlike the plane of the sky, the reference frame along the line of sight is unlikely to have an instrumental systematic drift, since each of the spectra are calibrated against $\mathrm{OH}$ lines (see $\S 3.2$ ). However, it is possible that there is a residual RV offset between the LSR and the S0-2 dynamical center. The Sun's peculiar motion with respect to the LSR along the line of sight might differ from the assumed $10 \mathrm{~km} \mathrm{~s}^{-1}$; that is, the practical realization of the LSR is not on a circular orbit around the Galactic center as might occur due the bar potential or to the spiral perturbations, so that the average velocity of stars in the solar vicinity might have a (small) net radial component. Alternatively, the dynamical center of S0-2 could differ from the dynamical center of the Galaxy as determined at the Sun's (i.e., LSR's) distance, as might result from the presence of an intermediate mass black hole companion. From the model fit, the implied motion of the LSR along the line-of-sight with respect to S0-2's dynamical center is $-20 \pm 33 \mathrm{~km} \mathrm{~s}^{-1}$, which is consistent with no net motion. While no significant motion is detected in $V_{x}, V_{y}$, or $V_{z}$, the $3 \sigma$ upper limits for the magnitudes of all three are comparable to one another in our 13-parameter model $\left(48,30\right.$, and $119 \mathrm{~km} \mathrm{~s}^{-1}$, respectively). Since there are no direct constraints on these quantities that can improve these limits, we have allowed them to be fully free parameters. However, if we assume that the black hole is stationary with respect to the Galaxy, we also need to consider the case of $V_{z}$ set to zero. ${ }^{13}$

\subsection{Point Mass Plus Extended Mass Distribution Analysis}

Limits on an extended mass distribution within S0-2's orbit are derived by assuming that the gravitational potential consists of a point mass and an extended mass distribution, and allowing for a Newtonian precession of the orbits (see, e.g., Rubilar \& Eckart 2001). In order to do this, we use the orbit-fitting procedure described in Weinberg et al. (2005) and adopt an extended mass distribution that has a power-law density profile $\rho(r)=\rho_{0}\left(r / r_{0}\right)^{-\gamma}$. This introduces two additional parameters to the model: the normalization of the profile and its slope $\gamma$. The total enclosed mass is then given by

$$
M(<r)=M_{\mathrm{bh}}+M_{\mathrm{ext}}\left(<r_{0}\right)\left(\frac{r}{r_{0}}\right)^{3-\gamma},
$$

where we quote values for the normalization $M_{\text {ext }}\left(<r_{0}\right)$ at $r_{0}=$ $0.01 \mathrm{pc}$, corresponding to the characteristic scale of the orbit. Figure 13 shows the constraint on $M_{\text {ext }}(<0.01 \mathrm{pc})$ and $\gamma$ from a fit to the astrometric and RV measurements for S0-2. The $99.7 \%$ confidence upper bound on the extended mass is $M_{\text {ext }}(<0.01 \mathrm{pc}) \simeq$ $3-4 \times 10^{5} M_{\odot}$ and has only a weak dependence on $\gamma$.

Mouawad et al. (2005) report a similar upper bound on the extended mass in fits to the orbit of S0-2. Their analysis differs only slightly from that presented here in that it forces the focus to be at the inferred radio position of Sgr $\mathrm{A}^{*}$, assumes a Plummer model mass distribution, and is based on data presented in Eisenhauer et al. (2003). Similarly, Zakharov et al. (2007) use an order of magnitude analysis to show that if the total mass of the extended matter enclosed within the S0-2 orbit is $\gtrsim 10^{5} M_{\odot}$, then it would produce a detectable apocenter shift $\Delta \phi \gtrsim 10$ mas (see also $\S 3.2$

13 Allowing for the uncertainty in the LSR in $V_{z}\left( \pm 2 \mathrm{~km} \mathrm{~s}^{-1}\right.$; Gould 2004) produces results that are not distinguishable from those reported for the $V_{z}=0$ case. 

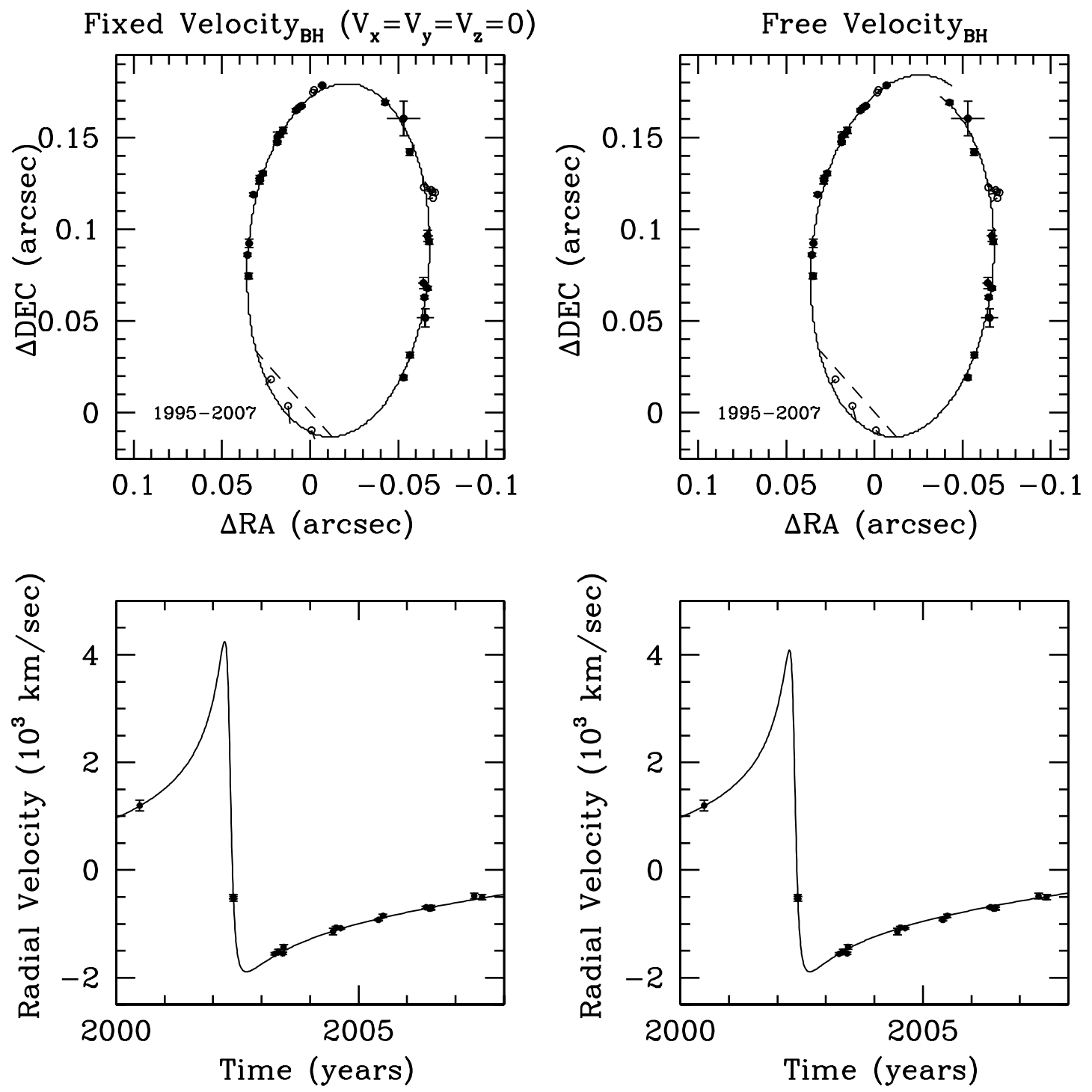

FIG. 9.-Best fit to the astrometric and RV data, assuming a Keplerian orbital model. The filled points were included in the formal fit, while the open points are measurements that are excluded due to source confusion. Uncertainties are plotted on all points, except the open/excluded points (here the uncertainties are comparable to the size of the points) for clarity. Left: To compare with what has been done in the past to estimate $R_{0}$, we show the fit to the data with a 10-parameter model, which includes the black hole's mass $\left(M_{\mathrm{bh}}\right)$, distance $\left(R_{0}\right)$, and location in the plane of the sky $\left(X_{0}, Y_{0}\right)$ as free parameters and which fixes the black hole's three-dimensional velocity $\left(V_{x}, V_{y}, V_{z}\right)$ to zero. This results in a $\chi^{2} /$ dof $\sim 1.4$. Right: The data are better reproduced by a 13-parameter model, which includes the black hole's mass $\left(M_{\mathrm{bh}}\right)$, distance $\left(R_{0}\right)$, location in the plane of the sky $\left(X_{0}, Y_{0}\right)$, and three-dimensional velocity $\left(V_{x}, V_{y}, V_{z}\right)$ as free parameters, and results in a $\chi^{2} /$ dof $\sim 0.97$. Adding these extra free parameters, and in particular $V_{z}$, increases the uncertainties in the black hole's properties by a factor of 2 .

in Weinberg et al. 2005). Hall \& Gondolo (2006) fit the total measured mass concentration $M(<r)$ to the data given in Ghez et al. (2005a), assuming a power-law density profile, and obtain an upper bound of $\approx 10^{5} M_{\odot}$ between 0.001 and $1 \mathrm{pc}$.

The surface brightness of stars as a function of projected radius from $\mathrm{Sgr} \mathrm{A}^{*}$ is well measured down to a radius of $\sim 0.5^{\prime \prime}$ $(\sim 0.02 \mathrm{pc})$. With an assumed constant mass-to-light ratio, the inferred stellar mass distribution between this inner radius and an outer radius of $10^{\prime \prime}$ is consistent with (Genzel et al. 2003b; see also Schödel et al. 2007):

$$
M_{*}(<r)=\left(6 \times 10^{5} M_{\odot}\right)\left(\frac{r}{0.4 \mathrm{pc}}\right)^{1.6} .
$$

Extrapolating this profile down to a radius of 0.01 pc gives an enclosed mass $M_{*}(<0.01 \mathrm{pc}) \approx 1-2 \times 10^{3} M_{\odot}$. Furthermore, theoretical estimates of the density of cold dark matter halo particles suggest that $\sim 1000 M_{\odot}$ of dark matter might reside in the inner
$0.01 \mathrm{pc}$ of the Galactic center (Gondolo \& Silk 1999; Ullio et al. 2001; Merritt et al. 2002; Gnedin \& Primack 2004). Likewise, the mass contribution from a cluster of stellar remnants, as predicted by Morris (1993) and Miralda-Escudé \& Gould (2000), is expected to be $\sim 1000 M_{\odot}$ within 0.01 pc. Unfortunately, these estimates are all smaller than the current upper bound by a factor of $\approx 100$. Measurements of stellar orbits with a next generation large telescope are, however, expected to be sensitive to an extended mass distribution of magnitude $M_{*}(<0.01 \mathrm{pc}) \approx 10^{3} M_{\odot}$ (Weinberg et al. 2005).

\section{DISCUSSION}

Orbit modeling of astrometric and radial velocity measurements of short-period stars provides a direct estimate of the Milky Way's central black hole mass and distance. Our analysis of S0-2's orbit yields a black hole mass of $M_{\mathrm{bh}}=4.1 \pm 0.6 \times 10^{6} M_{\odot}$ and distance of $R_{0}=8.0 \pm 0.6 \mathrm{kpc}$, if nothing is assumed about the black hole's intrinsic motion. If we assume that the black hole 

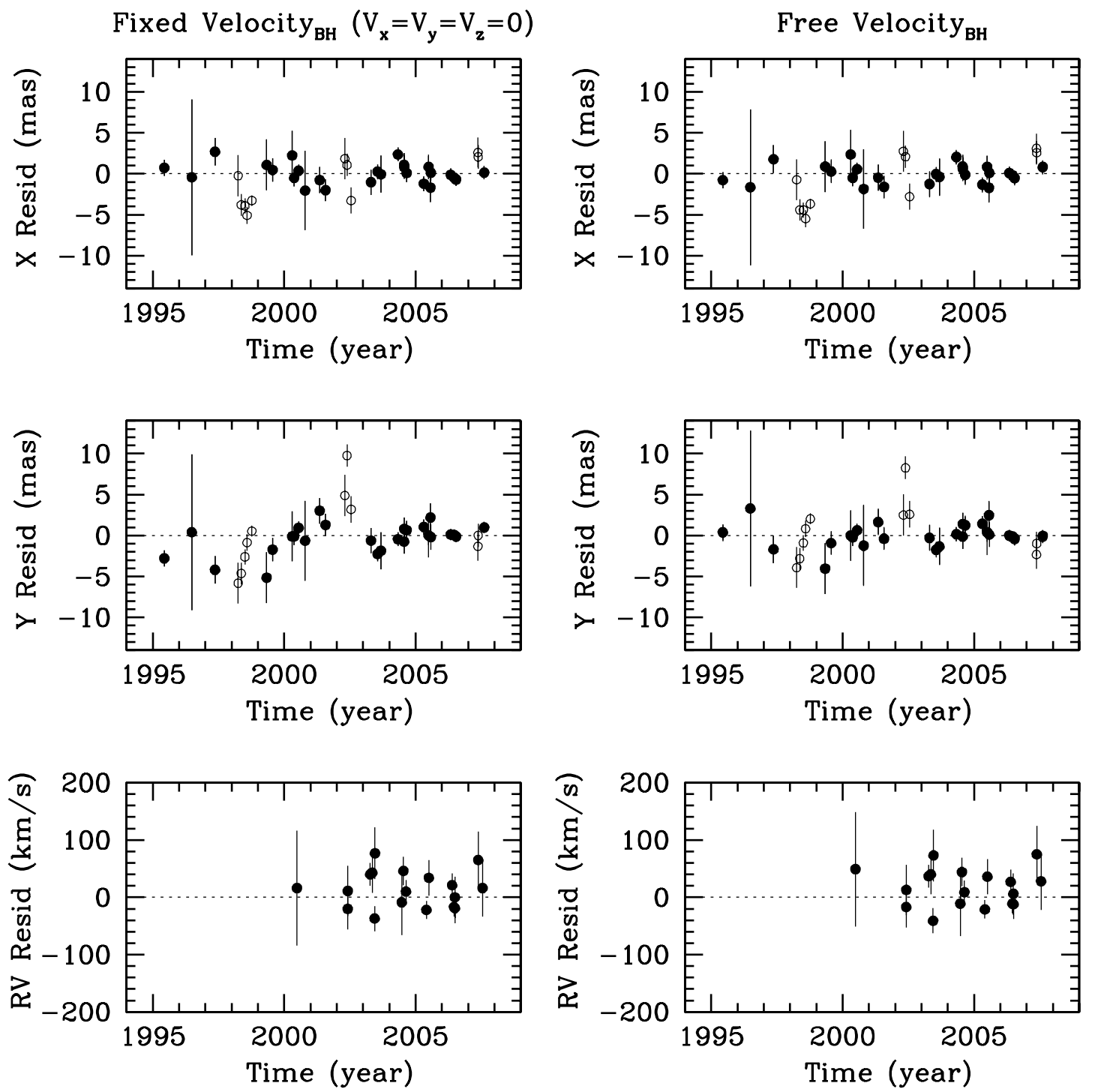

FIg. 10.- Residuals to the best-fit Keplerian orbital models shown in Fig. 9. The filled points were included in the formal fit, while the open points are measurements that are excluded due to source confusion.

has no intrinsic motion relative to the central stellar cluster (i.e., no massive companion), but still allow for systematics in the reference frames, then we obtain $M_{\mathrm{bh}}=4.5 \pm 0.4 \times 10^{6} M_{\odot}$ and distance of $R_{0}=8.4 \pm 0.4 \mathrm{kpc}$. This study shows that there are three systematic errors that must be accounted for to obtain accuracy in estimates of orbital parameters and this leads to larger uncertainties than have been reported in the past. First, since a dominant source of systematic error in the data set appears to be source confusion (see $\S \S 3$ and 4), we use only data from the brightest short orbital period star, S0-2, and only those measurements that are not confused with other known sources. Second, the motion of the black hole relative to the measurements' reference frame should be left as a free parameter, to account for both any possible intrinsic motion of the black hole as well as systematics in the astrometric or spectroscopic reference frames. Third, while Sgr A*-IR is detected with a precise position in deep LGSAO images, this finding appears to be biased; therefore, the position of the black hole should be treated as a free parameter in the fits in spite of the temptation to reduce the degrees of freedom with this detection. Because these systematics were not incorporated into earlier simultaneous estimates of $M_{\mathrm{bh}}$ and $R_{0}$ from the orbit of S0-2, the uncertainties in these initial studies were sig- nificantly underestimated; Eisenhauer et al. (2003, 2005), who do not account for the first two systematics, obtain $M_{\mathrm{bh}}=3.6 \pm 0$. $3 \times 10^{6} M_{\odot}$ and $R_{0}=7.6 \pm 0.3 \mathrm{kpc}$. Ghez et al. (2005a) used S0-2, S0-16, and S0-19 simultaneously, and allowed $V_{x}$ and $V_{y}$ to be free parameters, to derive a mass at fixed $R_{0}$ of $3.7 \pm 0.2 \times$ $10^{6}\left(R_{0} / 8 \mathrm{kpc}\right)^{3} M_{\odot}$, which was pulled down by the two astrometrically biased fainter stars, while Ghez et al. (2003) obtained a mass estimate of $4.1 \pm 0.6 \times 10^{6}\left(R_{0} / 8 \mathrm{kpc}\right)^{3} M_{\odot}$ from S0-2 alone. If we ignore the first two effects in model fits to our data, as was done by Eisenhauer et al. (2003, 2005; the only other work to estimate $R_{0}$ from orbits), we obtain a poor-quality fit ( $\chi^{2} /$ dof $=$ 2.0), uncertainties that are a factor of 2 smaller, and somewhat higher values than what we report in Table $5\left(M_{\mathrm{bh}}=4.7 \pm 0.3 \times\right.$ $10^{6} M_{\odot}$ and $\left.R_{0}=8.6 \pm 0.2 \mathrm{kpc}\right)$. The removal of biased astrometric points dominates the shift in the black hole's mass and distance to lower values in our analysis. This is somewhat surprising as this would suggest that similar removal of biased points might lower the Eisenhauer et al. (2005) results. However the biases may differ, as their early astrometric data measurements were made at 3 times lower angular resolution. An astrometric reference frame drift could also explain this effect, since $V_{x}$ and $V_{y}$ were held fixed in their analysis. The addition of $V_{z}$ as a free parameter dominates 


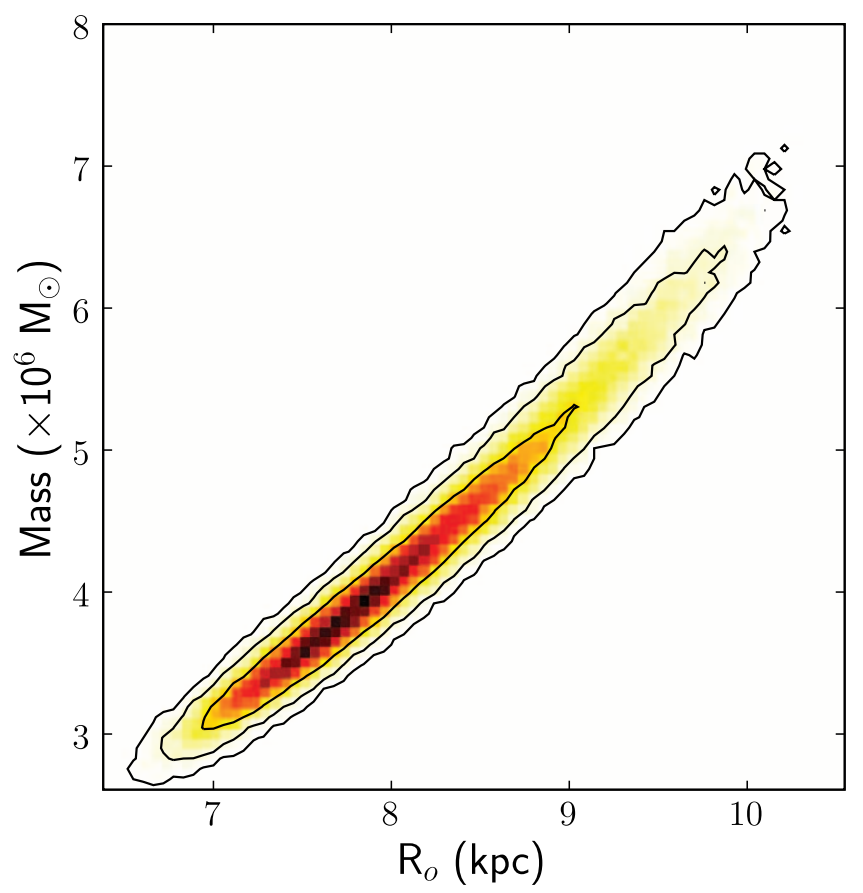

FIG. 11.-Correlation of the estimated black hole's mass and distance. The density of solutions from the MC simulations are shown as a color image, with the contours marking the $68 \%, 95 \%$, and $99.7 \%$ confidence limits. While mass and distance are well determined from the orbit of S0-2, they are not independent quantities. The exact scaling depends on the relative impact of the astrometric and radial data on the model fits. Currently, the inferred mass scales with the inferred distance as $M \propto R_{0}^{1.8}$.

the resulting uncertainties. In summary, in order to get an accurate measure of $M_{\mathrm{bh}}$ and $R_{0}$ from modeling of the short period orbits at the Galactic center, it is critical to account for the three sources of systematics described above.

The black hole mass measured here from a stellar orbit is larger than the $\sim 2-3 \times 10^{6} M_{\odot}$ inferred from using projected mass esti- mators, which rely on measured velocity dispersions (e.g., Eckart \& Genzel 1997; Genzel et al. 1997, 2000; Ghez et al. 1998; see also Chakrabarty \& Saha 2001). This difference most likely arises from the assumptions intrinsic to the use of projected mass estimators. In particular, the projected mass estimators are based on the assumption that the entire stellar cluster is measured, which is not the case for the early proper-motion studies, as their fields of view were quite small $(r \sim 0.1 \mathrm{pc})$. Such pencil-beam measurements can lead to significant biases (see discussions in Haller \& Melia 1996; Figer et al. 2003). An additional bias can arise if there is a central depression in the stellar distribution, such as that suggested by Figer et al. (2003). These biases can introduce factors of 2 uncertainties in the values of the enclosed mass obtained from projected mass estimates and thereby account for the difference between the indirect mass estimate from the velocity dispersions and the direct mass estimate from the orbital model fit to S0-2's kinematic data.

A higher mass for the central black hole brings our Galaxy into better agreement with the $M_{\mathrm{bh}}-\sigma$ relation observed for nearby galaxies (e.g., Ferrarese \& Merritt 2000; Gebhardt et al. 2000; Tremaine et al. 2002). For a bulge velocity dispersion that corresponds to that of the Milky Way $\left(\sim 103 \mathrm{~km} \mathrm{~s}^{-1}\right.$; Tremaine et al. 2002), the $M_{\mathrm{bh}}-\sigma$ relationship from Tremaine et al. (2002) predicts a black hole mass of $9.4 \times 10^{6} M_{\odot}$, which is a factor of 5 larger than the value of the Milky Way's black hole mass used by these authors $\left(1.8 \times 10^{6} M_{\odot}\right.$ from Chakrabarty \& Saha 2001). The black hole mass presented here of $4.1 \pm 0.6 \times 10^{6} M_{\odot}$ brings the Milky Way more in line with this relationship. With one of the most accurate and lowest central black hole masses, the Milky Way is, in principle, an important anchor for the $M_{\mathrm{bh}}-\sigma$ relationship. However, the velocity dispersion of the Milky Way is much more uncertain than that of other nearby galaxies. Therefore, our revised mass has only modest impact on the coefficients of the $M_{\mathrm{bh}}-\sigma$ relation.

Revision of the central black hole's mass and distance can also, in principle, impact our understanding of the structure within our galaxy both on small and large scales. On the large scale, if we assume that the black hole is located at the center of our Galaxy,

TABLE 5

Orbital Elements for S0-2 and the Implied Black Hole Properties

\begin{tabular}{|c|c|c|}
\hline Parameter & $V_{z}=0$ Case $^{\mathrm{a}}$ & $V_{z}$ Unconstrained Case \\
\hline Distance $\left(R_{0}\right)(\mathrm{kpc})^{\mathrm{b}}$. & $8.36 \pm_{0.44}^{0.30}$ & $7.96 \pm_{0.70}^{0.57}$ \\
\hline Period $(P)(\mathrm{yr}) \ldots \ldots \ldots \ldots \ldots$ & $15.78 \pm 0.35$ & $15.86 \pm_{0.45}^{0.10}$ \\
\hline 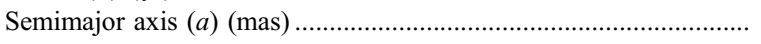 & $124.4 \pm_{3.3}^{2.4}$ & $126.5 \pm_{5.0}^{1.8}$ \\
\hline 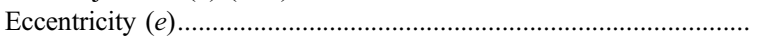 & $0.8866 \pm 0.0059$ & $0.8904 \pm_{0.0075}^{0.0051}$ \\
\hline 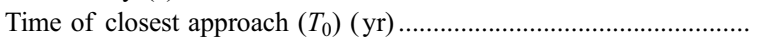 & $2002.3358 \pm_{0.0093}^{0.0065}$ & $2002.342 \pm 0.010$ \\
\hline 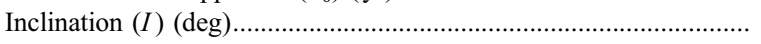 & $135.3 \pm 1.3$ & $134.6 \pm 1.3$ \\
\hline 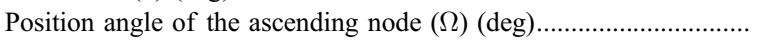 & $225.9 \pm 1.3$ & $226.44 \pm_{1.4}^{0.71}$ \\
\hline 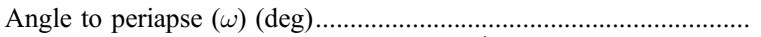 & $65.18 \pm 1.2$ & $66.0 \pm 1.7$ \\
\hline$X$ dynamical center $\left(X_{0}-X_{\text {Sgr A }}{ }^{*}\right.$-radio $)(\text { mas })^{\mathrm{b}, \mathrm{c}}$. & $0.95 \pm_{1.4}^{0.46}$ & $1.49 \pm_{0.87}^{1.1}$ \\
\hline 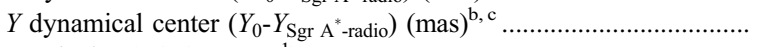 & $-4.8 \pm_{1.6}^{2.4}$ & $-5.4 \pm 2.0$ \\
\hline 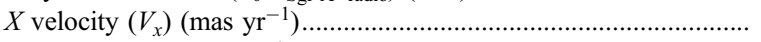 & $-0.40 \pm 0.25$ & $-0.47 \pm_{0.33}^{0.12}$ \\
\hline 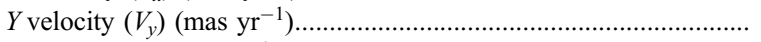 & $0.39 \pm_{0.18}^{0.09}$ & $0.36 \pm 0.12$ \\
\hline$Z$ velocity $\left(V_{z}\right)\left(\mathrm{km} \mathrm{s}^{-1}\right) \ldots \ldots \ldots \ldots \ldots \ldots$ & $\ldots$ & $-20 \pm \pm_{37}^{29}$ \\
\hline 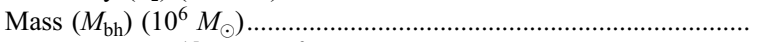 & $4.53 \pm_{0.55}^{0.34}$ & $4.07 \pm_{0.78}^{0.52}$ \\
\hline 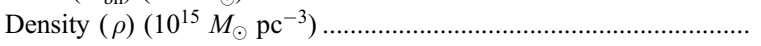 & $5.83 \pm_{0.97}^{0.28}$ & $6.3 \pm_{1.4}^{0.56}$ \\
\hline Periapse distance $\left(R_{\min }\right)(\mathrm{mpc}) .$. & $0.570 \pm 0.037$ & $0.535 \pm_{0.071}^{0.049}$ \\
\hline
\end{tabular}

NoтE.-Parameters below the horizontal line are derived from those above the line and are provided for convenience.

${ }^{a}$ Allowing for the uncertainty in the LSR in $V_{z}\left( \pm 2 \mathrm{~km} \mathrm{~s}^{-1}\right.$; Gould 2004) produces results that are not distinguishable from those reported above for the $V_{z}=0$ case.

b The reference time for the position of the black hole, when the velocity is a free parameter, is J2000.0.

${ }^{c}$ Uncertainties in the position of Sgr $\mathrm{A}^{*}$-radio are not incorporated into the uncertainties of $X_{0}$ and $Y_{0}$. 


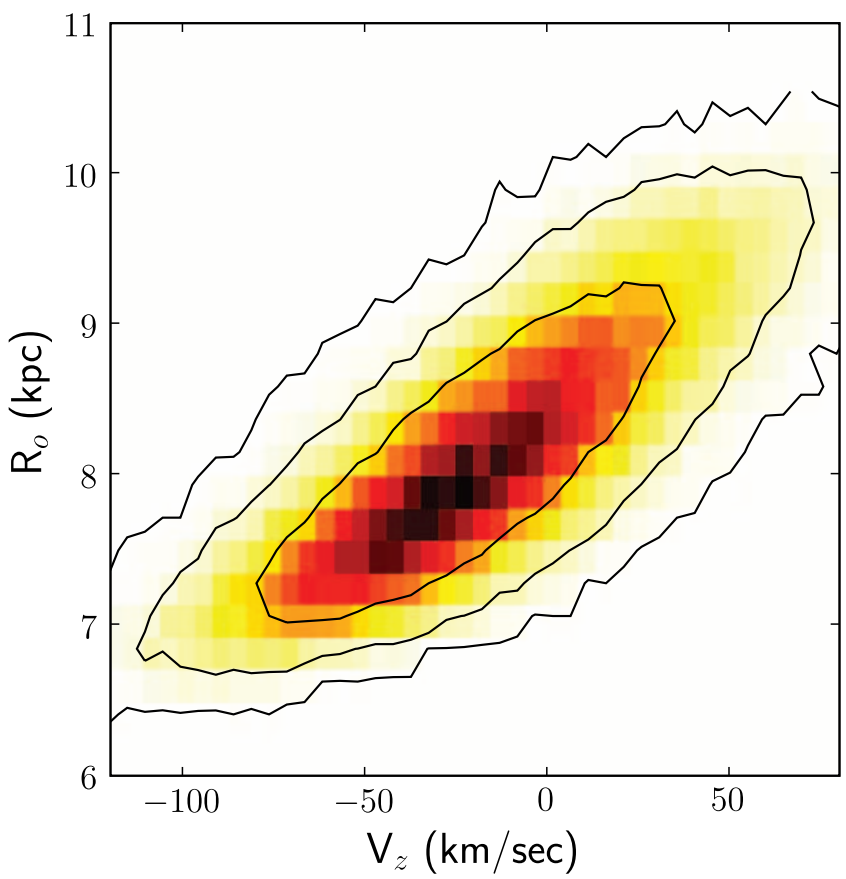

FIG. 12.-Correlation of the estimated black hole's distance and line-of-sight velocity $\left(V_{z}\right)$ from our 13-parameter model fit. $V_{z}$ dominates the uncertainties in $R_{0}$ and consequently $M_{\mathrm{bh}}$. Priors on $V_{z}$ can reduce the uncertainties in $R_{0}$ by a factor of 2. All previous studies have set $V_{z}$ to zero, which implicitly assumes that there are no massive companions to our Galaxy's central supermassive black hole and that the local standard of rest is perfectly known.

then its distance provides a measure of $R_{0}$. Its value from this study is consistent with the IAU recommended value of $8.5 \mathrm{kpc}$ as well as the value of $8.0 \pm 0.5 \mathrm{kpc}$ suggested by Reid (1993), based on a "weighted average" 14 of all prior indirect measurements of $R_{0}$. Combining the value for $R_{0}$ from this study with the proper motion of $\mathrm{Sgr} \mathrm{A}^{*}$ along the direction of Galactic longitude measured with VLBA in the radio quasar reference frame (Reid \&

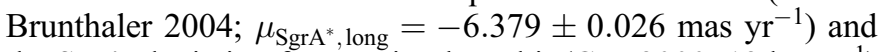
the Sun's deviation from a circular orbit (Cox 2000; $12 \mathrm{~km} \mathrm{~s}^{-1}$ ) in the direction of Galactic rotation, we obtain an estimate of the local rotation speed, $\theta_{0}$, of $229 \pm 18 \mathrm{~km} \mathrm{~s}^{-1}$, which is statistically consistent with other measurements; these include a value of $222 \pm 20 \mathrm{~km} \mathrm{~s}^{-1}$ from the review of Kerr \& Lynden-Bell (1986) and $270 \mathrm{~km} \mathrm{~s}^{-1}$ derived by Méndez et al. (1999) from the absolute proper motions of $\sim 30,000$ stars in the Southern Proper-Motion survey. As two of the fundamental Galactic constants, $R_{0}$ and $\theta_{0}$ are critical parameters for axisymmetric models of the Milky Way. Under the assumption that the stellar and gas kinematics within our Galaxy are well measured, the values of $R_{0}$ and $\theta_{0}$ determine the mass and shape of the Milky Way (Olling \& Merrifield 2000, 2001). Of particular interest is the value of the short-to-long axis ratio of the dark matter halo, $q$, as it offers a valuable opportunity to distinguish between different cosmological models. As Olling $\&$ Merrifield (2001) demonstrate, the uncertainty in $q$ for the Milky Way is dominated by the large uncertainties in $R_{0}$ and $\theta_{0}$. While our uncertainties in $R_{0}$ are currently too large to constrain $q$, future precision measurements of $R_{0}$ through stellar orbits may be able to do so and could thereby possibly distinguish between various dark matter candidates (Olling \& Merrifield 2001).

\footnotetext{
14 Consensus value with consensus errors.
}

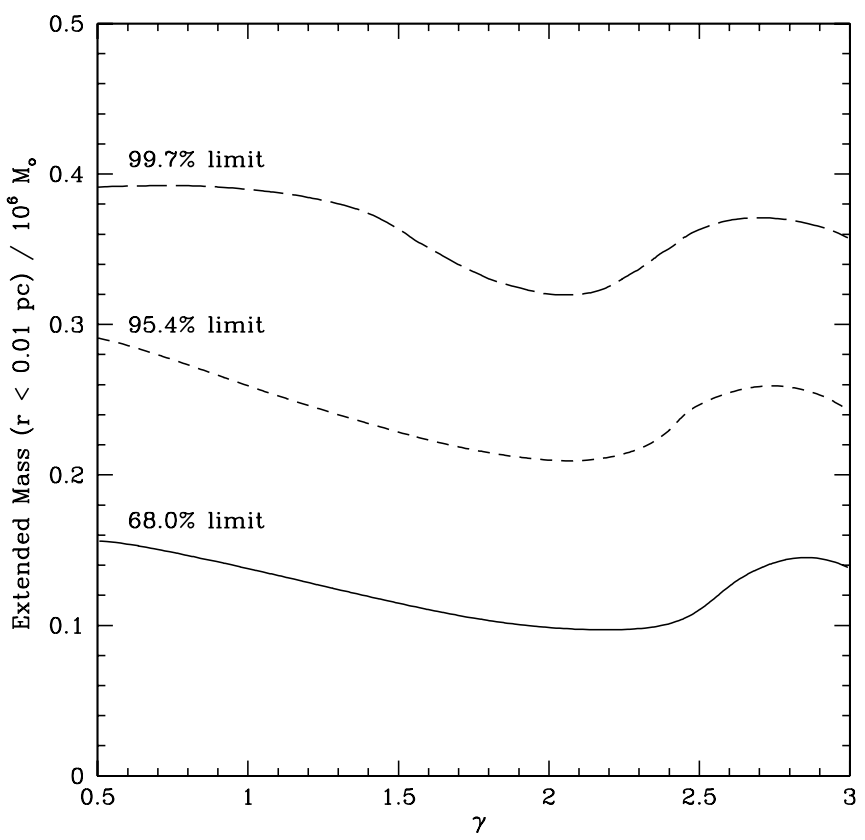

FIG. 13.- - Limits on the amount of mass in an extended distribution contained within S0-2's apoapse distance. The three lines correspond to the $68.3 \%, 95.4 \%$, and $99.7 \%$ upper-bound confidence limits. The $99.7 \%$ confidence upper bound of $3-4 \times 10^{5} M_{\odot}$ is a fairly weak function of the slope of the assumed power-law mass profile. Simple models of the stellar distribution suggest $M_{\text {ext }}(<0.01 \mathrm{pc}) \sim 10^{3} M_{\odot}$, a factor of $\sim 100$ smaller than the current measurement uncertainty.

Closer to the black hole, knowing its mass and distance from the Sun improves our ability to study the kinematics of stars within its sphere of influence. Much less kinematic information is needed to determine the orbital parameters for stars whose motion is dominated by the gravitational influence of the central black hole; for instance, with only measurements of a star's position, velocity, and acceleration in the plane of the sky along with a single line of sight velocity, a complete orbital solution can be derived once the black hole's mass and distance are well constrained. Improved constraints on the central black hole's properties and their degeneracies, as presented here, along with improved astrometry, has allowed us to derive orbital information for individual stars at much larger galactocentric distances. With these measurements, in Lu et al. $(2006,2008)$, we test for the existence and properties of the young stellar disk(s), proposed by Levin \& Beloborodov (2003) and Genzel et al. (2003b) from a statistical analysis of velocities alone. The direct use of individual stellar orbits out beyond a radius of $1^{\prime \prime}$ reveals only one, relatively thin disk of young stars ( Lu et al. 2008).

On an even smaller scale, the mass and distance of the black hole set the magnitude and timescale for various relativistic effects. Given estimated Keplerian orbital elements for stars at the Galactic center, we expect to be able to measure their stellar orbits with sufficient precision in upcoming years to detect the Roemer time delay, the special relativistic transverse Doppler shift, the general relativistic gravitational redshift, and the prograde motion of periapse (e.g., Weinberg et al. 2005; Zucker \& Alexander 2007). These effects will most likely be measured with S0-2 first, as it has the shortest orbital period $(P=15 \mathrm{yr})$, is quite eccentric $(e=0.89)$, and, as one of the brighter stars $\left(K_{\mathrm{S} 0-2}=14 \mathrm{mag}\right)$, it can be measured with the greatest astrometric and spectroscopic accuracy. The radial velocity signatures of the first three effects are expected to be comparable to each other and will impart a $\sim 200 \mathrm{~km} \mathrm{~s}^{-1}$ deviation at closest approach (Zucker \& Alexander 
2007), when the star is predicted to have a line of sight velocity of $-2500 \mathrm{~km} \mathrm{~s}^{-1}$ based on our updated Keplerian model. This effect is large compared to the radial velocity precision $\left(\sim 20 \mathrm{~km} \mathrm{~s}^{-1}\right)$. Likewise, the expected apoapse center shift for S0-2, $\Delta s=$ $\left(6 \pi G M_{\mathrm{bh}}\right) / R_{0}\left[(1-e) c^{2}\right]=0.9$ mas (see, e.g., Weinberg 1972; Weinberg et al. 2005), is an order of magnitude larger than our current measurement precision $\left(\sigma_{\text {pos }} \sim 0.1\right.$ mas). Improved adaptive optics systems on existing telescopes and larger telescopes (see Weinberg et al. 2005) will improve the sensitivity to the predicted apocenter shift. To put this measurement into context with existing tests of general relativity, it is useful to note that one of the strongest constraints on general relativity to date comes from the Hulse-Taylor binary pulsar, PSR 1913+16, which has a relativistic parameter at periapse, $\Gamma=r_{\mathrm{sch}} / r_{\text {periapse }}$, of only $5 \times$ $10^{-6}, \simeq 3$ orders of magnitude smaller than that of S0-2 (Taylor \& Weisberg 1989; Zucker \& Alexander 2007). The stars at the Galactic center are therefore probing an unexplored regime of gravity in terms of the relativistic object's mass scale and compactness.

Precession from general relativistic effects also influences the timescale for resonant relaxation processes close to the black hole (see, e.g., Rauch \& Tremaine 1996; Hopman \& Alexander 2006). When precession from general relativity dominates over that from the extended mass distribution, the resonant relaxation timescale is proportional to $M_{\mathrm{bh}}^{2} \times\left(J_{\mathrm{LSO}} / J\right)^{2} \times P$, where $J$ and $J_{\mathrm{LSO}}$ are the orbital angular momenta for the orbit of interest and at the last stable circular orbit around the black hole, respectively, and $P$ is the orbital period. For a given semimajor axis and accounting for the linear mass dependence of $\left(J_{\mathrm{LSO}} / J\right)^{2}$, this results in a $M_{\mathrm{bh}}^{5 / 2}$ dependency. Thus, the higher black hole mass inferred from this study increases the timescale over which the black hole's loss cone would be replenished in the regime where general relativity dominates. For the regime in which the extended mass distribution dominates, the resonant relaxation timescale scales only as $M_{\mathrm{bh}}^{1 / 2}$. A higher black hole mass also implies a longer period for the innermost stable circular orbit. If the central black hole is nonspinning, the innermost stable circular orbit has a period of $31 M_{\mathrm{bh}} /\left(4.1 \times 10^{6} M_{\odot}\right)$ minutes. Periodicities on shorter timescales, such as the putative quasi-periodic oscillation (QPO) at 20 minutes (Genzel. et al. 2003a; Eckart et al. 2006; Bélanger et al. 2006) have been interpreted as arising from the innermost stable circular orbit of a spinning black hole. At the present mass, the spin would have to be 0.6 of its maximal rate to be consistent with the possible periodicity. However, it is important to caution that other mechanisms can give rise to such short periodicities, such as a standing wave pattern recently suggested by Tagger \& Melia (2006). Furthermore, claims of a QPO in Sgr A* have been called into question; Do et al. (2008) find that the near-IR temporal power spectrum of Sgr $\mathrm{A}^{*}$ is statistically consistent with pure red noise, such as might be caused by disk instabilities or intermittent jet fluctuations, and Bélanger et al. (2008) reach a similar conclusion for the X-rays variations.

\section{CONCLUSIONS}

The short orbital period star S0-2 has been intensively studied astrometrically (1995-2007) and spectroscopically (2000-2007) with the W. M. Keck 10 m telescopes. Fits of a Keplerian orbit model to these data sets, after removing data adversely affected by source confusion, result in estimates of the black hole's mass and distance of $4.1 \pm 0.6 \times 10^{6} M_{\odot}$ and $8.0 \pm 0.6 \mathrm{kpc}$, respectively. While the current analysis is dominated by $11 \mathrm{yr}$ of astrometric measurements that have $\sim 1.2$ mas uncertainties, the LGSAO over the last 3 yr have positional uncertainties that are an order of magnitude smaller (100-200 $\mu$ as). With higher Strehl ratios and more sensitivity, LGSAO measurements are also less affected by source confusion; this is especially important for the closest approach measurements, which have to contend with source confusion from the variable source Sgr A*-IR. Following S0-2 for another $10 \mathrm{yr}$ should result in the measurement of the Sun's peculiar motion in the direction of the Galactic center from the orbit of S0-2 with a precision of a few kilometers per second and $1 \%$ measurement of $R_{0}$. At this precision, the measurement of $R_{0}$ is of particular interest because it could reduce the uncertainty in the cosmic distance ladder.

We thank the staff of the Keck Observatory, especially Joel Aycock, Randy Campbell, Al Conrad, Jim Lyke, David LeMignant, Chuck Sorensen, Marcos Van Dam, Peter Wizinowich, and director Taft Armandroff for all their help in obtaining the new observations. We also thank Brad Hanson, Leo Meyer, and Clovis Hopmann for their constructive comments on the manuscript, and the referee, Rainer Schodel, for his helpful suggestions. Support for this work was provided by NSF grant AST 04-06816 and the NSF Science and Technology Center for Adaptive Optics, managed by the University of California, Santa Cruz (AST 98-76783), and the LevineLeichtman Family Foundation. The W. M. Keck Observatory is operated as a scientific partnership among the California Institute of Technology, the University of California and the National Aeronautics and Space Administration. The Observatory was made possible by the generous financial support of the W. M. Keck Foundation. The authors wish to recognize and acknowledge the very significant cultural role and reverence that the summit of Mauna Kea has always had within the indigenous Hawaiian community. We are most fortunate to have the opportunity to conduct observations from this mountain.

Facilities: Keck:II (NIRC2, NIRSPEC, OSIRIS), Keck:I(NIRC)

\section{APPENDIX A}

\section{CLUSTER REFERENCE FRAME}

All positional measurements from the individual images $\left(X^{\prime}, Y^{\prime}\right)$ are transformed with a full first-order polynomial to a common reference system $(X, Y)$, which we refer to as the cluster reference frame (see Ghez et al. 1998, 2000, 2005a; Lu et al. 2008). The transformations are derived by minimizing the net displacements, allowing for proper motions, of all the coordinate reference stars (see $\S 3.1$ ) relative to their positions in a common reference image (ref), which for this study is the 2004 July LSGAO image. Specifically, we minimize the following sum over the coordinate reference stars $(s)$ :

$$
D=\sum_{s}^{N_{\text {stars }}}\left(\Delta X_{s, e}^{2}+\Delta Y_{s, e}^{2}\right) / W_{s, e}
$$



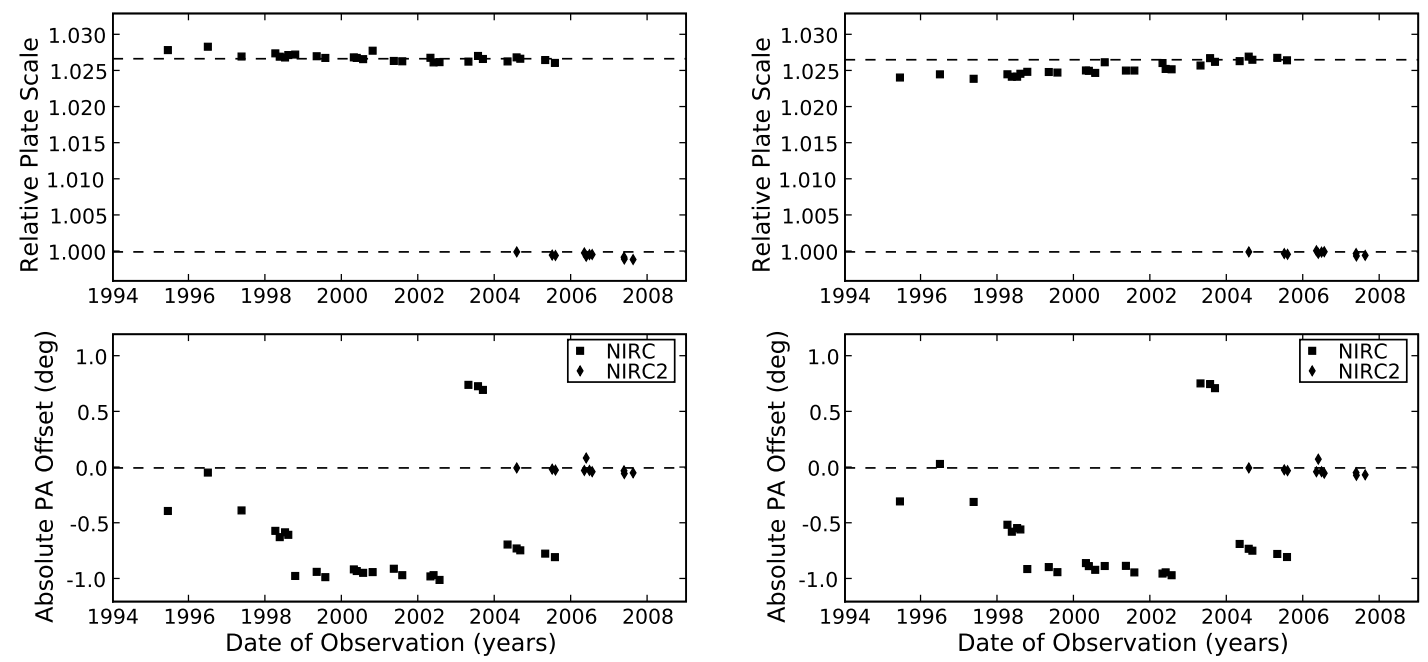

FIG. 14.- Plate scale (top) and the position angle (bottom) over time for all data sets aligned using a set of stars that excludes (left) and includes (right) the known young stars. The plate scale is relative to the plate scale in the reference epoch of 2004 July LGS. The P.A. is the absolute position angle offset from the value reported in the NIRC and NIRC2 instrument headers. Once the young stars are excluded, the estimated plate scales for NIRC (squares) and NIRC2 (diamonds) are very stable, approximately $0.05 \%$ and $0.03 \%$ (rms), respectively, over multiple years. NIRC shows several systematic jumps in the position angle relative to the value reported in the image headers, which is most likely a result of instrument or telescope changes.

where $\Delta X_{s, e}=X_{s, \text { ref }}+V_{x_{s}} \times\left(t_{e}-t_{\text {ref }}\right)-X_{s, e}, \Delta Y_{s, e}=Y_{s, \text { ref }}+V_{y_{s}} \times\left(t_{e}-t_{\text {ref }}\right)-Y_{s, e}$, and $W_{s, e}=\sigma_{\Delta X_{s, e}^{\prime}}+\sigma_{\Delta Y_{s, e}^{\prime}}$, and where $X$ and $Y$ are expressed as the following function of the measured positions $X^{\prime}$ and $Y^{\prime}$ for each epoch $(e)$ :

$$
\begin{gathered}
X_{e}=a_{0_{e}}+a_{1_{e}} \times X_{e}^{\prime}+a_{2_{e}} \times Y_{e}^{\prime}, \\
Y_{e}=b_{0_{e}}+b_{1_{e}} \times X_{e}^{\prime}+b_{2_{e}} \times Y_{e}^{\prime} .
\end{gathered}
$$

The coefficients for the reference epoch are fixed to $a_{1_{\text {ref }}}=b_{2_{\text {ref }}}=1$ and $a_{0_{\text {ref }}}=a_{2_{\text {ref }}}=b_{0_{\text {ref }}}=b_{1_{\text {ref }}}=0$ and the coefficients for the remaining epochs $\left(a_{0_{e}}, a_{1_{e}}, a_{2_{e}}, b_{0_{e}}, b_{1_{e}}\right.$, and $\left.b_{2_{e}}\right)$ come from the minimization of $D$. Because of degeneracies between coordinate transformations and proper motions of the coordinate reference stars, the net displacement is minimized in two steps. First, $D$ is minimized with the proper motions $\left(V_{x}\right.$ and $V_{y}$ ) of the coordinate reference stars set to zero in order to obtain preliminary transformation coefficients. Using these initial coefficients, we transform all the positional measurements to a common coordinate system and fit a linear motion model to them in order to derive a first pass estimate of the proper motions. Second, $D$ is minimized again, using the preliminary proper motions and holding them fixed, while the final transformation coefficients are derived.

This procedure produces proper motions for the coordinate reference stars that have no significant mean motion. We therefore conclude that the resulting cluster reference frame is stable and free of significant systematics. This procedure is also used to check the stability of the combined effects of the camera systems and the coordinate reference stars. By carrying out transformations that allow for only translation, rotation, and a scale change, we examine the apparent stability of the camera's pixel scale and angle relative to that recorded in the header. Figure 14 (left-hand panels) shows that the relative pixel scales for the cameras are stable to within $0.053 \%$ (rms) over the time baseline of this study and that the uncertainty in the angle relative to the header P.A. is dominated by inaccuracies in the header value (most of the jumps correspond to times when the camera is known to have been opened for engineering purposes). This also provides a measure of the resampled NIRC pixel scale relative to the NIRC2 pixel scale $(1.0269 \pm 0.0005)$ and an absolute NIRC pixel scale of $20.46 \pm 0.01$ mas pixel $^{-1}$ when combined with the absolute NIRC2 pixel scale from Appendix C. The right-hand panels of Figure 14 display the results of the same exercise but using a set of coordinate reference stars that includes the known young stars; the clear systematic trend in the relative pixel scales demonstrates the importance of removing this set of stars with known net rotation from the coordinate reference star list.

\section{APPENDIX B}

\section{NIRC2 GEOMETRIC OPTICAL DISTORTIONS}

Relative stellar positions from the deep LGSAO images have accuracies $(\sim 0.2 \mathrm{mas})$ that are an order of magnitude smaller than the currently available optical distortion map for NIRC2. ${ }^{15}$ Since LGSAO/NIRC2 data were obtained with four different setups (e.g., centerings and/or position angles on the sky), imperfections in the optical distortion corrections can introduce 1-2 mas systematics, if unaccounted for, into the relative positions of S0-2 (and S0-16). We therefore introduce two steps into our analysis to correct for this effect. First, we add, in quadrature, an additional 0.88 mas to all the LGSAO positional measurements of the coordinate reference stars, such that the proper motions and hence coordinate transformations are not biased. The magnitude of this term is derived by finding the value that reduced the average offset of these LGSAO points from the linear proper-motion fits, which exclude these points, from 5 to $1 \sigma$. Second, we derive explicit correction terms for the local optical distortions for S0-2 and S0-16 positions in each of LGSAO epochs not obtained with the same set up as the reference image (2004 July), using the orbits of five "calibration" stars (S0-3, S0-7, S0-19, S0-26, and S0-27) that are

\footnotetext{
${ }^{15}$ See http://www2.keck.hawaii.edu/inst/nirc2/preship_testing.pdf.
} 


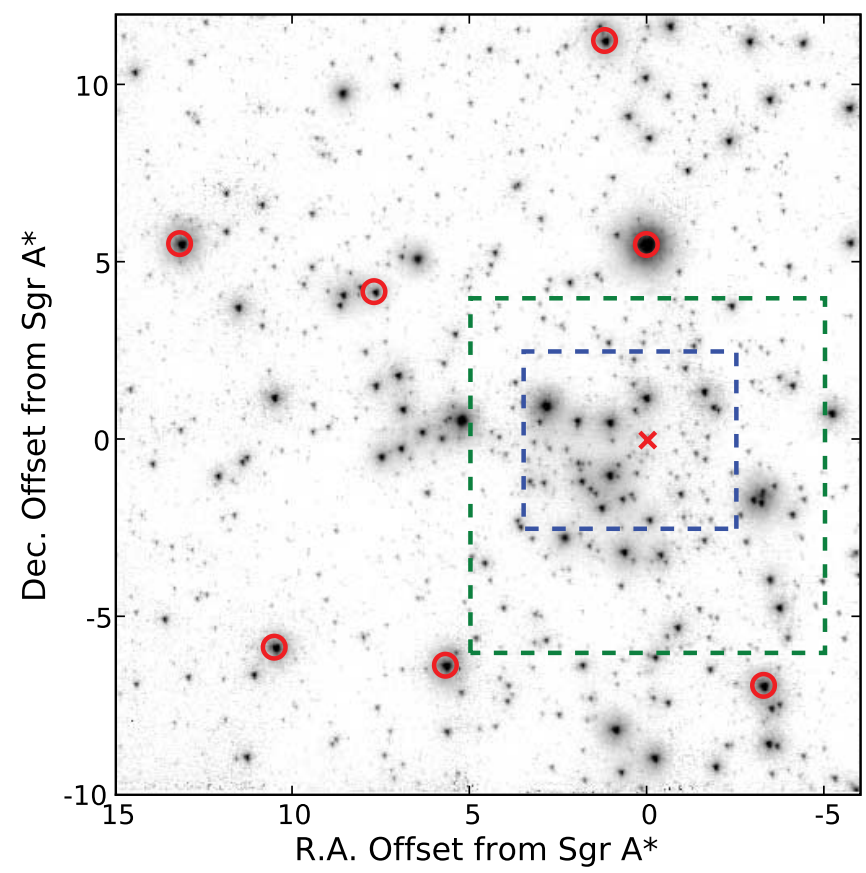

Fig. 15.- Infrared mosaic measuring the positions of the SiO masers. The seven masers, whose radio positions are well measured by Reid et al. (2007) and which are used to establish an absolute reference frame, are circled. Dotted lines depict the outline of example LGSAO (green) and speckle (blue) images in which the short-period stars are measured and placed in the cluster reference frame. Since the masers sparsely sample the area of interest, only low-order polynomials are used to calibrate the cluster reference frame (i.e., pixel scale, orientation, and position of Sgr $\mathrm{A}^{*}$-radio).

within $0.5^{\prime \prime}$ of S0-2. These terms are obtained by first using only the speckle data, which are distortion calibrated with respect to the reference image (2004 July/LGSAO; see Lu et al. 2008), the reference image (taken with setup 1), and the one other LGSAO image taken with the same setup as the reference image to solve for the orbits of the five calibration stars. For each LGSAO epoch not included in these fits, the average offsets of these five stars' aligned measurements from their predicted location is used to characterize the residual distortions for that image (relative to the reference image) at the position of S0-2 and S0-16 and the standard deviation of the offsets provide an estimate of the uncertainties in these values. Setup 3 is the only LGSAO observational configuration, other than that used for the reference image, used in multiple epochs. From the measurements with setup 3, it can be seen that the rms of their estimated bias terms ( 0.24 mas) is smaller than the uncertainty in each bias term estimated from the rms of the five stars ( $\sim 0.67 \mathrm{mas})$. This suggests that the bias terms are relatively static (see also Appendix A) and that their uncertainties are dominated by our uncertainties in the stellar orbits (and possible structure in the distortion on scales $<0.5^{\prime \prime}$ ). We therefore derive an average bias correction value and uncertainty for each setup. The final bias terms, which range in value between 1.6 and 2.6 mas, are added to the LGSAO positional measurements made with setups $2-4$ in the analysis presented in $\S 4$ and their uncertainties are added in quadrature with the uncertainties associated with centroiding and coordinate transformation; this bias term as already been incorporated into the values and uncertainties reported in Table 3. Correlations in the bias corrections for setup 3 are applied and accounted for in the Monte Carlo simulations described in $\S 4$.

\section{APPENDIX C}

\section{ABSOLUTE ASTROMETRY}

An absolute astrometric reference frame for the Galactic center was established from radio observations of seven SiO masers ( Reid et al. 2003, 2007). Relative measurements in the infrared were tied to the absolute frame by observing, in the infrared, the red giant stars that are

TABLE 6

Maser Properties

\begin{tabular}{|c|c|c|c|c|c|c|c|c|}
\hline$K$ magnitude........ & 9.1 & 7.7 & 9.5 & 9.3 & 11.3 & 10.2 & 8.9 & $\ldots$ \\
\hline$X$ position $(\operatorname{arcsec})$ & 5.679 & 0.032 & -3.264 & 10.484 & 7.684 & 1.209 & 13.139 & $\ldots$ \\
\hline 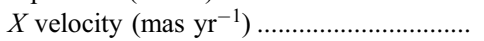 & 3.06 & -0.58 & -1.06 & 2.00 & 0.04 & -1.96 & -1.61 & . \\
\hline 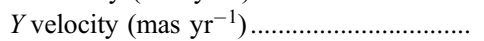 & 2.11 & -3.52 & -2.70 & -5.29 & -2.09 & -5.68 & -0.75 & $\ldots$ \\
\hline 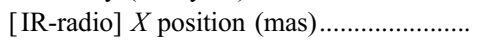 & $3.7 \pm 5.2$ & $3.5 \pm 7.2$ & $11.6 \pm 7.3$ & $0.6 \pm 5.9$ & $-3.9 \pm 3.4$ & $-1.9 \pm 5.5$ & $-5.3 \pm 5.2$ & $1.2 \pm 5.7$ \\
\hline 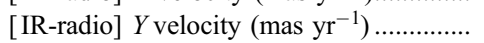 & $0.4 \pm 0.8$ & $-1.7 \pm 0.6$ & $0.7 \pm 1.0$ & $-0.5 \pm 2.6$ & $-1.5 \pm 0.3$ & $0.0 \pm 0.4$ & $-3.5 \pm 1.3$ & $-0.9 \pm 1.5$ \\
\hline
\end{tabular}

Notes.-Maser positional uncertainties and differences are averaged over the the maser epochs: $2005.495,2006.336,2007.612$. The values in the last column are the average and standard deviation of the values for the individual masers. 
TABLE 7

NIRC2 Absolute Astrometry

\begin{tabular}{|c|c|c|c|}
\hline Parameter & 2005.495 & 2006.336 & 2007.612 \\
\hline Plate scale $\left(\right.$ mas pixel $\left.{ }^{-1}\right)$ & $9.963 \pm 0.005$ & $9.964 \pm 0.004$ & $9.961 \pm 0.006$ \\
\hline 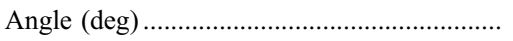 & $0.12 \pm 0.02$ & $0.13 \pm 0.02$ & $0.14 \pm 0.02$ \\
\hline
\end{tabular}

NoTE.-The final values for the NIRC2 plate scale and the position angle of the NIRC2 columns with respect to North Sgr $\mathrm{A}^{*}$ are averages over these values and their errors.

the source of the maser emission (Figure 15 and Table 6). Observations were taken in 2005 June, 2006 May, and 2007 August using LGSAO/NIRC2 (see $\S 2$ ) with $10.86 \mathrm{~s}$ integrations in the $K^{\prime}$ band, each composed of 60 co-added $0.181 \mathrm{~s}$ exposures in order to avoid saturating the bright masers. A nine-position dither box pattern was used to construct a $22^{\prime \prime} \times 22^{\prime \prime}$ mosaic with two exposures at each position for the 2005 mosaic and three exposures at each position for the 2006 and 2007 mosaics. The individual frames for each data set were cleaned, undistorted, and then registered and mosaicked using the IRAF xregister and drizzle routines. Subset mosaics were also created with only one exposure at each position and were used to derive centroiding uncertainties. StarFinder was run on the resulting mosaicked images to extract stellar positions and uncertainties from the RMS error of the subset mosaics. Centroiding errors were typically on the order of 1.4 mas. This yields an IR star list for each epoch with positions in NIRC2 pixel coordinates.

The radio maser positions were propagated forward using velocities from Reid et al. (2007) to create a radio maser star list at the epoch of each of the above IR mosaics. Uncertainties in these propagated radio positions are, on average, $\sim 1.4$ mas. For each epoch, the IR maser star list was aligned to the radio mosaic star list, which resulted in a new IR mosaic star list in the absolute astrometric reference frame with $\mathrm{Sgr}^{*}$-radio at the origin. This alignment process used only four independent parameters (a global pixel scale, a rotation, and an origin in the $x$ - and $y$-directions) to transform between the NIRC2 coordinate system of the IR mosaics to the absolute coordinate system of the radio masers. While using higher order polynomial transformations reduce the residual offsets positions from Sgr A* between the infrared and radio measurements, we conservatively chose to use this low-order transformation to capture within the uncertainties the possible impact of systematics, such as uncorrected residual camera distortions and differential atmospheric refraction. This is particularly important given the sparse sampling of masers across the region of interest (see Fig. 15). Uncertainties in the transformation to absolute coordinates, which were determined with a half-sample bootstrap Monte Carlo simulation of 100 iterations, where each iteration uses only half the stars in each star list, were added in quadrature to the infrared centroiding uncertainties to produce a final uncertainties in the infrared absolute positions of the masers. After the transformation to absolute coordinates, the absolute value of the offsets between the positions of the masers relative to $\mathrm{Sgr} \mathrm{A}^{*}$ measured in the infrared and radio are on average 0.8 and $0.8 \sigma$, or equivalently, 5.7 and 5.7 mas in the $x$ - and $y$-direction, respectively (see Table 6); we take this to be our uncertainty in the position of Sgr $\mathrm{A}^{*}$ radio in the infrared maser mosaic. Likewise, the transformations between the infrared and radio reference frame yields a plate scale of $9.963 \pm 0.005$ mas pixel ${ }^{-1}$ and a position angle offset for NIRC2 of $0.13^{\circ} \pm 0.02^{\circ}$. Each of the three infrared maser mosaics yields comparable results (see Table 7). Uncertainties in the absolute positions in the infrared reference frame are dominated by residual optical distortions, which are amplified by the large dithers necessary to construct the mosaics.

A comparison of the maser's proper motions as measured in the radio and the infrared provides an estimate of how accurately we can transform our relative measurements into a reference frame in which $\mathrm{Sgr} \mathrm{A}^{*}$-radio is at rest and the orientation is set by background quasars (Reid et al. 2007). The absolute infrared proper motions of the masers, as well as all other stars detected in the infrared maser mosaics, were derived by fitting a linear model to the positions as a function of time from the three IR maser star lists that were separately aligned to the radio reference frame. Because the alignment uncertainties are dominated by residual distortion and therefore correlated across epochs for a given maser, this source of uncertainty is not included in the linear proper-motion modeling. The differences in the proper motions measured in the radio and in the infrared have an average value of $0.6 \pm 0.4$ and $-0.9 \pm 0.6 \mathrm{mas} \mathrm{yr}^{-1}$ in the $x$ - and $y$-directions, respectively, where the uncertainties are the standard deviation of the mean. Therefore, at present, it is not possible to use these measurements to eliminate possible drifts in the cluster reference frame as the source of any apparent $V_{x}$ or $V_{y}$ from the orbital fits of S0-2 (see $\S 4.1)$.

The relative astrometry measurements presented in $\S 3$ were transformed into this absolute reference frame through a set of infrared stars we designated as infrared absolute astrometric standards. Absolute astrometric standards were defined to be those stars that (1) are de-

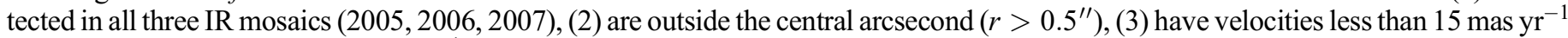
and velocity errors less than 5 mas $\mathrm{yr}^{-1},(4)$ have reasonable velocity fits $\left(\chi^{2} /\right.$ dof $\left.<4\right)$, and $(5)$ are brighter than $K=15$. With absolute kinematics for 158 stars within $5^{\prime \prime}$, we solve for a four-parameter transformation model by comparing the relative positions in the reference epoch image, which are in instrumental pixel coordinates, and the estimated absolute coordinates for that epoch, which are in arcseconds relative to the position of $\mathrm{Sgr} \mathrm{A}^{*}$. Since all other epochs are aligned to this reference epoch, positional measurements for all stars in all epochs are easily transformed into absolute coordinates. While uncertainties in the absolute infrared reference frame dominate the final absolute positional uncertainties relative to Sgr $\mathrm{A}^{*}$-radio, they are a negligible source of uncertainty for the orbital analysis.

\section{REFERENCES}

Bélanger, G., Terrier, R., de Jager, O. C., Goldwurm, A., \& Melia, F. 2006, J.

Phys. Conf. Ser., 54, 420

2008, ApJ, submitted

Chakrabarty, D., \& Saha, P. 2001, AJ, 122, 232

Cox, A. N. 2000, Allen's Astrophysical Quantities (4th ed.; New York: AIP)

Dehnen, W., \& Binney, J. J. 1998, MNRAS, 298, 387

Diolaiti, E., Bendinelli, O., Bonaccini, D., Close, L., Currie, D., \& Parmeggiani, G. 2000, A\&AS, 147, 335
Do, T., Ghez, A. M., Morris, M. R., Yelda, S., Lu, J. R., Hornstein, S., \& Matthews, K. 2008, ApJ, in press (arXiv:0810.0446)

Eckart, A., \& Genzel, R. 1997, MNRAS, 284, 576

Eckart, A., Genzel, R., Ott, T., \& Schödel, R. 2002, MNRAS, 331, 917

Eckart, A., Schödel, R., Meyer, L., Trippe, S., Ott, T., \& Genzel, R. 2006, A\&A, 455, 1

Eisenhauer, F., Schödel, R., Genzel, R., Ott, T., Tecza, M., Abuter, R., Eckart, A., \& Alexander, T. 2003, ApJ, 597, L121 
Eisenhauer, F., et al. 2005, ApJ, 628, 246

Ferrarese, L., \& Merritt, D. 2000, ApJ, 539, L9

Figer, D. F., et al. 2003, ApJ, 599, 1139

Fragile, P. C., \& Mathews, G. J. 2000, ApJ, 542, 328

Freitag, M., Amaro-Seoane, P., \& Kalogera, V. 2006, ApJ, 649, 91

Gebhardt, K., et al. 2000, ApJ, 539, L13

Genzel, R., Eckart, A., Ott, T., \& Eisenhauer, F. 1997, MNRAS, 291, 219

Genzel, R., Pichon, C., Eckart, A., Gerhard, O. E., \& Ott, T. 2000, MNRAS, 317,348

Genzel, R., Schödel, R., Ott, T., Eckart, A., Alexander, T., Lacombe, F., Rouan, D., \& Aschenbach, B. 2003a, Nature, 425, 934

Genzel, R., et al. 2003b, ApJ, 594, 812

Gezari, S., Ghez, A. M., Becklin, E. E., Larkin, J., McLean, I. S., \& Morris, M. 2002, ApJ, 576, 790

Ghez, A. M., Klein, B. L., Morris, M., \& Becklin, E. E. 1998, ApJ, 509, 678

Ghez, A. M., Morris, M., Becklin, E. E., Tanner, A., \& Kremenek, T. 2000, Nature, 407, 349

Ghez, A. M., Salim, S., Hornstein, S. D., Tanner, A., Lu, J. R., Morris, M., Becklin, E. E., \& Duchêne, G. 2005a, ApJ, 620, 744

Ghez, A. M., et al. 2003, ApJ, 586, L127 2004, ApJ, 601, L159 2005b, ApJ, 635, 1087

Gnedin, O. Y., \& Primack, J. R. 2004, Phys. Rev. Lett., 93, 061302

Gondolo, P., \& Silk, J. 1999, Phys. Rev. Lett., 83, 1719

Gould, A. 2004, ApJ, 607, 653

Hall, J., \& Gondolo, P. 2006, Phys. Rev. D, 74, 063511

Haller, J. W., \& Melia, F. 1996, ApJ, 464, 774

Hanson, M. M., Conti, P. S., \& Rieke, M. J. 1996, ApJS, 107, 281

Hopman, C., \& Alexander, T. 2006, ApJ, 645, 1152

Hornstein, S. D. 2007, Ph.D. thesis, UCLA

Hornstein, S. D., Matthews, K., Ghez, A. M., Lu, J. R., Morris, M., Becklin, E. E., Rafelski, M., \& Baganoff, F. K. 2007, ApJ, 667, 900

Jaroszyński, M. 1998, Acta Astron., 48, 653 1999, ApJ, 521, 591

Kerr, F. J., \& Lynden-Bell, D. 1986, MNRAS, 221, 1023

Kraniotis, G. V. 2007, Class. Quantum Grav., 24, 1775

Larkin, J., et al. 2006, NewA Rev., 50, 362

Levin, Y., \& Beloborodov, A. M. 2003, ApJ, 590, L33

Lu, J. R., Ghez, A. M., Hornstein, S. D., Morris, M., Matthews, K., Thompson, D. J., \& Becklin, E. E. 2006, J. Phys. Conf. Ser., 54, 279 2008, ApJ, in press (arXiv:0808.3818)

Majewski, S. R., Law, D. R., Polak, A. A., \& Patterson, R. J. 2006, ApJ, 637, L25

Matthews, K., Ghez, A. M., Weinberger, A. J., \& Neugebauer, G. 1996, PASP, 108,615

Matthews, K., \& Soifer, B. T. 1994, Ap\&SS, 190, 239
McLean, I. S., Graham, J. R., Becklin, E. E., Figer, D. F., Larkin, J. E., Levenson, N. A., \& Teplitz, H. I. 2000, Proc. SPIE, 4008, 1048

McLean, I. S., et al. 1998, Proc. SPIE, 3354, 566

Melia, F., \& Falcke, H. 2001, ARA\&A, 39, 309

Méndez, R. A., Platais, I., Girard, T. M., Kozhurina-Platais, V., \& van Altena, W. F. 1999, ApJ, 524, L39

Merritt, D., Milosavljević, M., Verde, L., \& Jimenez, R. 2002, Phys. Rev. Lett., 88,191301

Miralda-Escudé, J., \& Gould, A. 2000, ApJ, 545, 847

Morris, M. 1993, ApJ, 408, 496

Mouawad, N., Eckart, A., Pfalzner, S., Schödel, R., Moultaka, J., \& Spurzem, R. 2005, Astron. Nachr., 326, 83

Nikiforov, I. I. 2008, in Dynamics of Galaxies, in press (arXiv:0803.0825)

Olling, R. P., \& Merrifield, M. R. 2000, MNRAS, 311, 361 2001, MNRAS, 326, 164

Rafelski, M., Ghez, A. M., Hornstein, S. D., Lu, J. R., \& Morris, M. 2007, ApJ, 659,1241

Rauch, K. P., \& Tremaine, S. 1996, NewA, 1, 149

Reid, M. J. 1993, ARA\&A, 31, 345

Reid, M. J., \& Brunthaler, A. 2004, ApJ, 616, 872

Reid, M. J., Menten, K. M., Genzel, R., Ott, T., Schödel, R., \& Eckart, A. 2003, ApJ, 587, 208

Reid, M. J., Menten, K. M., Trippe, S., Ott, T., \& Genzel, R. 2007, ApJ, 659, 378

Rubilar, G. F., \& Eckart, A. 2001, A\&A, 374, 95

Salim, S., \& Gould, A. 1999, ApJ, 523, 633

Schödel, R., Ott, T., Genzel, R., Eckart, A., Mouawad, N., \& Alexander, T. 2003, ApJ, 596, 1015

Schödel, R., et al. 2002, Nature, 419, 694 2007, A\&A, 469, 125

Tagger, M., \& Melia, F. 2006, ApJ, 636, L33

Tremaine, S., et al. 2002, ApJ, 574, 740

Ullio, P., Zhao, H., \& Kamionkowski, M. 2001, Phys. Rev. D, 64, 043504

van Dam, M. A., et al. 2006, PASP, 118, 310

Weinberg, N. N., Milosavljević, M., \& Ghez, A. M. 2005, ApJ, 622, 878

Weinberg, S. 1972, Gravitation and Cosmology: Principles and Applications of the General Theory of Relativity (New York: Wiley)

Will, C. M. 2008, ApJ, 674, L25

Taylor, J. H., \& Weisberg, J. M. 1989, ApJ, 345, 434

Wizinowich, P. L., Acton, D. S., Lai, O., Gathright, J., Lupton, W., \& Stomski, P. J. 2000, Proc. SPIE, 4007, 2

Wizinowich, P. L., et al. 2006, PASP, 118, 297

Zakharov, A. F., Nucita, A. A., de Paolis, F., \& Ingrosso, G. 2007, Phys. Rev. D, 76,062001

Zucker, S., \& Alexander, T. 2007, ApJ, 654, L83 